\title{
Cortical and Subcortical Dysmetabolism Are Dynamic Markers of Clinical Disability and Course in Anti-LGI1 Encephalitis
}

Eero Rissanen, MD, PhD, Kelsey Carter, MSN, CNP, Steven Cicero, BS, John Ficke, BA, Marie Kijewski, DSc, Mi-Ae Park, PhD, Joseph Kijewski, BS, Emily Stern, MD, Tanuja Chitnis, MD, David Silbersweig, MD, Howard L. Weiner, MD, Chun K. Kim, MD, Jennifer Lyons, MD, Joshua P. Klein, MD, PhD, Shamik Bhattacharyya, MD, MS, and Tarun Singhal, MD, MBBS

Neurol Neuroimmunol Neuroinflamm 2022;9:e1136. doi:10.1212/NXI.0000000000001136

\section{Abstract}

\section{Background and Objectives}

This $\left[{ }^{18} \mathrm{~F}\right]$ fluorodeoxyglucose (FDG) PET study evaluates the accuracy of semiquantitative measurement of putaminal hypermetabolism in identifying anti-leucine-rich, glioma-inactivated-1 (LGI1) protein autoimmune encephalitis (AE). In addition, the extent of brain dysmetabolism, their association with clinical outcomes, and longitudinal metabolic changes after immunotherapy in LGI1-AE are examined.

\section{Methods}

FDG-PET scans from 49 age-matched and sex-matched subjects (13 in LGI1-AE group, 15 in non-LGI1-AE group, 11 with Alzheimer disease [AD], and 10 negative controls [NCs]) and follow-up scans from 8 patients with LGI1 AE on a median 6 months after immunotherapy were analyzed. Putaminal standardized uptake value ratios (SUVRs) normalized to global brain (P-SUVRg), thalamus (P/Th), and midbrain (P/Mi) were evaluated for diagnostic accuracy. SUVRg was applied for all other analyses.

\section{Results}

P-SUVRg, P/Th, and P/Mi were higher in LGI1-AE group than in non-LGI1-AE group, AD group, and NCs (all $p<0.05)$. P/Mi and P-SUVRg differentiated LGI1-AE group robustly from other groups (areas under the curve 0.84-0.99). Mediotemporal lobe (MTL) SUVRg was increased in both LGI1-AE and non-LGI1-AE groups when compared with NCs (both $p<$ 0.05). SUVRg was decreased in several frontoparietal regions and increased in pallidum, caudate, pons, olfactory, and inferior occipital gyrus in LGI1-AE group when compared with that in NCs (all $p<0.05)$. In LGI1-AE group, both MTL and putaminal hypermetabolism were reduced after immunotherapy. Normalization of regional cortical dysmetabolism associated with clinical improvement at the 6- and 20-month follow-up.

\section{Discussion}

Semiquantitative measurement of putaminal hypermetabolism with FDG-PET may be used to distinguish LGI1-AE from other pathologies. Metabolic abnormalities in LGI1-AE extend beyond putamen and MTL into other subcortical and cortical regions. FDG-PET may be used in evaluating disease evolution in LGI1-AE.

\author{
Correspondence \\ Dr. Singhal \\ tsinghal@bwh.harvard.edu
}

\section{MORE ONLINE}

(II) Class of Evidence Criteria for rating therapeutic and diagnostic studies

NPub.org/coe 


\section{Glossary}

$\left[{ }^{18} \mathrm{~F}\right]$ FDG $=\left[{ }^{18} \mathrm{~F}\right]$ fluorodeoxyglucose; $\mathrm{AAL}=$ automated anatomical labeling; $\mathrm{AD}=$ Alzheimer disease; $\mathrm{AE}=$ autoimmune encephalitis; AMPA = $\alpha$-amino-3-hydroxy-5-methyl-4-isoxazolepropionic acid; APS = antiphospholipid syndrome; AUC = area under the curve; $\mathbf{B W H}=$ Brigham and Women's Hospital; DMN = default mode network; FBDS = facio-brachial dystonic seizures; GABA = gamma-aminobutyric acid; $\mathbf{H E}=$ Hashimoto encephalopathy; $\mathbf{I Q R}=$ interquartile range; $\mathbf{L E}=$ limbic encephalitis; LGI1 = leucine-rich, glioma-inactivated-1; mRS = modified Rankin scale; $\mathbf{M T L}=$ mediotemporal lobe; NC = negative controls; $\mathbf{P} / \mathbf{M i}$ = putamen-to-midbrain ratio; $\mathbf{P} / \mathbf{T h}$ = putamen-to-thalamus ratio; $\mathbf{P}$-SUVRg = putaminal global brain normalized SUV ratio; ROC = receiver operating characteristic; ROI = regions of interest; $\mathbf{S S}=$ Sjögren syndrome; SUVR = standardized uptake value ratio.

\section{Classification of Evidence}

This study provides Class II evidence that semiquantitative measures of putaminal metabolism on PET can differentiate patients with LGI1-AE from patients without LGI1-AE, patients with $\mathrm{AD}$, or NCs.

Encephalitis associated with leucine-rich, glioma-inactivated1 (LGI1) protein autoantibodies is a form of autoimmune encephalitis $(\mathrm{AE})$ in which the autoantibodies targeting LGI1 protein inflict a cascade of inflammatory changes affecting synaptic function and causing subsequent neuronal damage., ${ }^{1,2}$ LGI1 protein bridges the presynaptic voltage-gated potassium channel (protein Kv1.1 and the postsynaptic $\alpha$-amino-3hydroxy-5-methyl-4-isoxazolepropionic acid (AMPA) receptor by interacting with the presynaptic ADAM23 and postsynaptic ADAM22 anchor molecules. ${ }^{2,3}$ Anti-LGI1 antibodies prevent the binding of LGI1 to ADAM22 and ADAM23, resulting in decreased density of both Kv1.1 and AMPA receptors and giving rise to neuronal hyperexcitability and reduced neuronal plasticity. ${ }^{4}$ LGI1-AE commonly affects middle-aged and elderly individuals, and the typical symptoms include subacute memory impairment, confusion, and seizures. ${ }^{3,5}$ The stereotypical facio-brachial dystonic seizures (FBDSs) are considered a diagnostic clue for LGI1-AE, ${ }^{6,7}$ although present only in approximately half of the patients. ${ }^{8,9}$

Early recognition, diagnosis, and rapidly started immunotherapy are necessary for improving prognosis in patients with LGI1AE. ${ }^{1,10,11}$ However, an initial diagnosis of LGI1-AE can be missed or considerably delayed in most patients because of a low clinical index of suspicion. ${ }^{12}$ LGI1-AE is overrepresented in elderly individuals (older than 60 years) when compared with other forms of $\mathrm{AE}$ and may be mistaken for Alzheimer disease $(\mathrm{AD})^{13}$ or other dementias. ${ }^{12}$ An initial MRI finding may indicate $\mathrm{AE}$ but requires further confirmation. ${ }^{5,6,8,9,14}$ Furthermore, standard inflammatory workup from CSF can be normal, ${ }^{5,15}$ and results from specific AE-associated autoantibody screening could take several days to return in clinical practice. ${ }^{16}$

PET imaging of brain metabolism with $\left[{ }^{18} \mathrm{~F}\right]$-fluorodeoxyglucose (FDG) may be more sensitive and specific than MRI in identifying patients with LGI1-AE. ${ }^{9}$ Putaminal and medial temporal hypermetabolism have been reported as the prominent metabolic abnormalities in anti-LGI1-AE ${ }^{9,17,18}$ but the accuracy of FDGPET in distinguishing patients with LGI1-AE from patients with other autoimmune encephalopathies and neurodegenerative conditions is not clear. Finally, apart from a few recent case series with a short follow-up duration, ${ }^{9,19}$ longitudinal metabolic changes and their clinical relevance in LGI1-AE has not been systematically studied.

The primary research questions of this study are as follows: (1) to evaluate the accuracy of semiquantitative brain FDGPET analysis in differentiating LGI1-AE group from nonLGI1-AE group, $\mathrm{AD}$ group, and from controls without known neurologic disease, (2) to determine the extent of dysmetabolism in cortical and subcortical regions beyond the previously reported putaminal and mediotemporal lobe (MTL) abnormalities, and (3) to evaluate longitudinal changes in brain metabolism after immunosuppressive treatment in LGI1-AE and their association with concurrent clinical disability and long-term clinical outcome.

\section{Methods}

\section{Participants}

A total of 57 FDG-PET scans in 49 individuals were identified retrospectively by performing a database search for patients who had undergone brain or whole body FDG-PET between 2008 and 2019 and based on individual clinicians' referrals. Encephalitis, limbic, autoimmune, LGI1, and encephalopathy were used as search terms to identify patients suspected of $A E$. Of the 55 individuals, 28 patients with $\mathrm{AE}$ fulfilling the Graus 2016 criteria $^{20}$ were identified as antibody-positive LGI1-AE group $(\mathrm{n}=13)$ or non-LGI1-AE group $(\mathrm{n}=15)$. Of the patients with LGI1-AE, 8 had a follow-up FDG-PET performed on a median 5.7 (interquartile range [IQR] 6.5) months after the baseline PET. Age-matched and sexmatched patients with diagnosis of probable $\mathrm{AD}(\mathrm{n}=11)$ were included as positive controls with an alternate, known primary brain disorder that can mimic LGI1. ${ }^{13}$ Similarly, a cohort of age-matched and sex-matched individuals without known primary neurologic or neuropsychiatric disease or structural brain abnormality, for whom a whole body 
FDG-PET (including brain) had been performed for a nonneurologic indication (treatment planning or restaging of cutaneous malignancies), were included as negative controls (NCs, $\mathrm{n}=10$ ).

The non-LGI1-AE group included 4 patients with antiNMDA receptor $A E$, 1 with anti-gamma-aminobutyric acid (GABA)-B receptor AE, 1 with anti-CASPR2-AE, 2 with anti-GAD65-AE, 1 with anti-Zic4/GAD65-AE, 1 with anti-Hu-AE, 3 with Hashimoto encephalopathy (HE), 1 with Sjögren syndrome (SS), and 1 with antiphospholipid syndrome (APS)-associated AE. AD diagnoses were confirmed by FDG-PET and neuropsychologic testing and/or CSF analyses (amyloid-beta-42, total tau, and phosphorylated tau). Demographics of the study subjects are presented in eTable 1 , links.lww.com/NXI/A695.

\section{Longitudinal Clinical Follow-up}

Clinical, MRI, and EEG data were extracted for all subjects in LGI1-AE and non-LGI1-AE groups. The level of clinical disability for those in LGI1-AE group at baseline (at first FDG-PET), short-term follow-up (at the time of follow-up FDG-PET), and long-term follow-up (at the latest clinical follow-up) was determined using modified Rankin scale (mRS). Disability at baseline was stratified into mild-tomoderate $(\mathrm{mRS} \leq 3)$ or into at least moderately severe ( $\mathrm{mRS}$ $>3$ ) because of the patients' high level of disability at that time. ${ }^{21}$ Clinical short-term and long-term outcomes were stratified into good (none-to-mild disability; $m R S \leq 2$ ) and poor (at least moderate disability; $\mathrm{mRS}>2$ ) outcomes based on $\mathrm{mRS}$ at respective time points. The median (range) time of the long-term clinical follow-up was 19.7 (0.7-41.0) months.

\section{Standard Protocol Approval}

This retrospective, investigator-initiated academic study was conducted at Brigham and Women's Hospital (BWH; Boston, MA). The study protocol was approved by the Institutional Review Board at BWH. Being a retrospective study using data acquired during routine clinical care, it was exempt from the requirement to obtain informed consent from study subjects.

\section{$\left[{ }^{18} \mathrm{~F}\right]$ FDG-PET Imaging and Image Analyses}

$\left[{ }^{18} \mathrm{~F}\right]$ FDG radiopharmaceutical production and FDG-PET imaging were performed according to BWH's standard clinical procedures. PMOD Neuro Tool (PNEURO) 3.8 platform (PMOD Technologies, Zurich, Switzerland) was used for processing and analyzing the brain FDG-PET images.

Segmentation of regions of interest (ROI) was performed with automated anatomical labeling (AAL) template within the PNEURO utilities suite. ${ }^{22}$ The mean standardized uptake values (SUVs) were extracted for putamen (average of right and left) and MTL (combined right and left averages of hippocampus, amygdala, and parahippocampus). SUVs were also obtained for other supratentorial and brainstem ROIs based on the AAL template.
For semiquantitative analyses, normalizations of SUVs from target region were performed for global brain (volumeweighted average SUV of all AAL template brain ROIs including white matter), thalamus (average SUV of right and left), and midbrain ROIs. Thus, the following FDG-PET indices were acquired for primary analyses: putaminal global brain normalized SUV ratio (P-SUVRg), putamen-to-thalamus ratio $(\mathrm{P} / \mathrm{Th})$, putamen-to-midbrain ratio $(\mathrm{P} / \mathrm{Mi}), \mathrm{MTL}-\mathrm{SUVRg}$, MTL/Th, and MTL/Mi for mediotemporal lobe. Both global and thalamic normalizations are standard techniques used routinely in brain FDG imaging. ${ }^{23-25}$ Global normalized SUVRgs were also obtained for the remaining AAL ROIs. Image analyses were performed blinded to the patients' disease severity ( $\mathrm{mRS}$ status).

\section{Statistical Methods}

Demographics were compared between groups using the Fisher exact and Kruskal-Wallis tests. Differences in FDGPET parameters between LGI1-AE, non-LGI1-AE, AD and NC groups were evaluated using the Kruskal-Wallis test with Bonferroni correction. For subgroup analyses, patients in non-LGI1 group were further grouped into patients with NMDA receptor $A E(n=4)$, other limbic encephalitis (LE)like-AEs (LE-like-AE: with GABA-B, CASPR2, anti-Hu, or GAD65-antibodies; $\mathrm{n}=6$ ), and LE-like-AE associated with other autoimmune systemic disorders (LE: SS, HE, and APS; $n=5$ ). Longitudinal changes in mRS scores in LGI1-AE group were evaluated using the Friedman Test. For the LGI1-AE subgroup with follow-up FDG-PET, changes in FDG uptake from baseline to follow-up were evaluated with the Wilcoxon signed rank test, and comparisons of FDG-PET indices at baseline and follow-up against NCs were performed using the Mann-Whitney $U$ test. Performance of the FDG-PET indices in differentiating LGI1-AE group from other groups was evaluated with receiver operating characteristic (ROC) curve analysis. A comparison of ROC area under the curve (AUC) between FDG-PET indices was performed with DeLong ${ }^{26}$ statistics. Using the ROC curve coordinates, cutoff values for optimal sensitivity and specificity for the FDG-PET indices were evaluated by identifying the highest Youden index ${ }^{27}(\mathrm{~J}=$ sensitivity + specificity -1 ). If 2 equal $\mathrm{J}$ values were observed, the one with higher sensitivity was chosen. Positive and negative likelihood ratios (LR+ and LR-) were calculated for the cutoff values. The $p$ values $<0.05$ were regarded statistically significant, unless stated otherwise. IBM SPSS Statistics, version 24.0 (IBM Corp., Armonk, NY) was used for all statistical analyses except for comparison of AUCs, where MedCalc Statistical software, version 19.5.2 (MedCalc Software Ltd, Ostend, Belgium) was used.

Statistical parametric mapping (SPM; SPM12 software, Wellcome Centre for Human Neuroimaging, London, UK) was used for voxel-wise evaluation of parametric SUVRg images for those with LGI1-AE. PET images were stereotactically normalized to the Montreal Neurologic Institute template. Differences in baseline SUVRg in LGI1-AE patients with good vs poor long-term outcome were compared with 
independent $t$ test. Paired $t$ test was applied to evaluate longitudinal metabolic changes from baseline to short-term follow-up PET in patients with different levels of disability at the follow-up PET. Parametric $t$ statistic maps visualizing voxels with significant correlations, group differences, and within-group changes were produced.

\section{Data Availability}

Any anonymized data not provided in this article will be shared on request from any qualified investigator for procedure and result replication purposes.

\section{Results}

\section{Clinical Characteristics}

Detailed clinical characteristics of those in LGI1-AE and non-LGI1-AE groups are listed in Tables 1 and 2. The median (range) time from symptom onset to the first $\left[{ }^{18} \mathrm{~F}\right] \mathrm{FDG}$ PET scan was $3.2(0.2-13.7)$ months for the LGI1-AE and 3.2 (0.4-36.6) months for the non-LGI1-AE groups $(p=0.786)$. Generalized tonic-clonic seizures were observed in 31\% (4/13) in the LGI1-AE and in 40\% (6/15) in the non-LGI1-AE groups $(p=0.705)$.

Among patients with LGI1-AE, 69\% (9/13) presented with unilateral or bilateral FBDSs, and cognitive impairment was observed in all subjects. Regarding clinical disability at the time of first FDG-PET, the median (range) mRS score was 4 (1-5) (Table 1).

\section{Conventional Imaging and EEG Findings}

On visual interpretation, all baseline FDG-PET scans in LGI1-AE and non-LGI1-AE groups were abnormal. In LGI1AE group, MRI findings showed either cortical, mesiotemporal, or putaminal hyperintensity in 8 of the $13(62 \%)$ cases, whereas an additional 3 cases showed nonspecific white matter abnormalities (Table 1). The rate of EEG abnormalities was not different between LGI1-AE and non-LGI1-AE groups $(8 / 12,67 \%$ vs $11 / 12,92 \% ; p=0.317)$. Similarly, there was no difference in the proportion of patients receiving immunosuppressive and antiseizure treatment (23\% vs $47 \%, p=$ $0.254 ; 69 \%$ vs $47 \%, p=0.276$, respectively) at the time of the first FDG-PET in LGI1-AE vs non-LGI1-AE groups. Further details regarding MRI and EEG abnormalities and treatment in both LGI1-AE and non-LGI1-AE groups are presented in Tables 1 and 2 .

\section{Putaminal Hypermetabolism in LGI1-AE}

Increased putaminal metabolic activity was observed in LGI1-AE group when compared with all other groups (i.e., non-LGI1-AE group, $\mathrm{AD}$ group, and NCs) with all the putaminal indices $(p<$ 0.001 for P-SUVRg, $p=0.002$ for $\mathrm{P} / \mathrm{Th}$, and $p<0.001$ for $\mathrm{P} /$ $\mathrm{Mi})$. On pair-wise comparisons, LGI1-AE group exhibited increased P-SUVRg, higher $\mathrm{P} / \mathrm{Th}$, and higher $\mathrm{P} / \mathrm{Mi}$ values when compared with non-LGI1-AE group $(+13.8 \%, p=0.014$ for P-SUVRg; $+14.1 \%, p=0.010$ for $\mathrm{P} / \mathrm{Th}$, and $+18.8 \%, p=0.001$ for P/Mi). Similarly, P-SUVRg, P/Th, and P/Mi were higher in
LGI1-AE group in comparison with NCs $(+19.8 \%, p<0.001$ for SUVRg; $+16.0 \%, p=0.004$ for $\mathrm{P} / \mathrm{Th}$, and $+14.6 \%, p=0.009$ for $\mathrm{P} / \mathrm{Mi}$ ) (Figure 1A, eTable 2, links.lww.com/NXI/A695). In non-LGI1-AE subgroup analyses, P-SUVRg and $\mathrm{P} / \mathrm{Mi}$ ratio were higher in LGI1-AE when compared with NMDA receptor $\mathrm{AE}$, and all 3 putaminal indices were higher in LGI1-AE when compared with other LE-like-AEs (eTable 3).

When compared with $\mathrm{AD}$ group, LGI-AE group demonstrated higher P-SUVRg, P/Mi, and P/Th-values, but only the differences in $\mathrm{P} / \mathrm{Mi}$ achieved statistical significance $(+15.7 \%$, $p=0.001$ for $\mathrm{P} / \mathrm{Mi} ;+12.7 \%, p=0.131$ for SUVRg, and $+9.5 \%$, $p=0.076$ for $\mathrm{P} / \mathrm{Th}$ ) (Figure 1A, eTable 2, links.lww.com/ NXI/A695). The ROI-based, semiquantitative findings of abnormal putaminal metabolism were consistent with individual visualization using parametric SUVRg images (Figure 1C). No differences were observed in the putaminal indices between patients with LGI1-AE with or without FBDS at the time of the initial FDG-PET.

\section{Mediotemporal Hypermetabolism in Both LGI1-AE and Non-LGI1-AE Groups}

There were no differences between LGI1-AE and non-LGI1AE groups or non-LGI1-AE subgroups in the MTL indices. However, abnormally increased MTL index values were seen in both LGI1-AE and non-LGI-AE groups when compared with NCs (Figure 1B, eTables 2-3, links.lww.com/NXI/A695).

Specifically, higher MTL-SUVRg, MTL/Th, and MTL/Mi values were observed in LGI1-AE group when compared with NCs $(+16.5 \%, p=0.008 ;+11.1 \%, p=0.048$ and $+10.2 \%, p=$ 0.033 , respectively). Similarly, in non-LGI1-AE vs NC comparison, MTL-SUVRg and MTL/Th values were higher in non-LGI1-AE group than those in NCs $(+14.7 \%, p=0.003$ and $+11.9 \%, p=0.016$, respectively).

When compared with $\mathrm{AD}$ group, both LGI1-AE and nonLGI1-AE groups exhibited higher MTL/Mi values (+12.0\%, $p$ $=0.001$ for LGI1 vs $\mathrm{AD}$ and $+10.5 \%, p=0.014$ for non-LGI1 vs $\mathrm{AD}$ ) (Figure 1B, eTable 2, links.lww.com/NXI/A695). No differences were observed in the MTL indices when comparing patients with LGI1-AE with or without FBDS at the time of the initial FDG-PET.

\section{Putamen-to-Midbrain Ratio Best Differentiates LGI1-AE Group From Other Groups}

On ROC analyses, all 3 putaminal indices provided significant differentiation for distinguishing patients with LGI1-AE from NCs, non-LGI1-AE group, and $\mathrm{AD}$ group (all $p<0.05$ ) (Figure 2A). There were no differences in the ROC AUCs between P-SUVRg, P/Mi, and P/Th values in any of the group comparisons $(p=\mathrm{ns}$ for all). However, when evaluated for optimal accuracy, P-SUVRg provided the best combination of sensitivity and specificity (manifesting as the highest absolute Youden J values at the index-specific cutoff values) of the 3 putaminal indices for the comparison of LGI1-AE group vs NCs, and P/Mi provided the highest absolute $\mathrm{J}$ values for 
Table 1 Demographic, Clinical, Antibody Testing, MRI, and EEG Characteristics of the LGI1-AE Group

\begin{tabular}{|c|c|c|c|c|c|c|c|c|}
\hline $\begin{array}{l}\text { Case } \\
\text { no }\end{array}$ & $\begin{array}{l}\text { Age } \\
\text { (y)l } \\
\text { sex }\end{array}$ & $\begin{array}{l}\text { LGI1- } \\
\text { antibody } \\
\text { test } \\
\text { result }\end{array}$ & $\begin{array}{l}\text { Clinical presentation before } \\
\text { first PET (mRS score at first } \\
\text { PET) }\end{array}$ & $\begin{array}{l}\text { Time from onset } \\
\text { to first PET (time } \\
\text { to follow-up PET) } \\
\text { (mo) }\end{array}$ & $\begin{array}{l}\text { MRI at the time } \\
\text { of first PET }\end{array}$ & $\begin{array}{l}\text { EEG at the time } \\
\text { of first PET }\end{array}$ & $\begin{array}{l}\text { Immunomodulatory } \\
\text { treatments }\end{array}$ & $\begin{array}{l}\text { Neurology follow-up time. } \\
\text { Clinical outcome (mRS), } \\
\text { other notable }\end{array}$ \\
\hline 1 & $\begin{array}{l}77 / \\
M\end{array}$ & $\begin{array}{l}\text { S-LGI1-Ab } \\
\text { positive }\end{array}$ & $\begin{array}{l}\text { R-dominant FBDS, cognitive } \\
\text { impairment (mRS 4). }\end{array}$ & $1.3(9.3)$ & Normal & $\begin{array}{l}\text { B/L cerebral } \\
\text { and } L>R \\
\text { temporal } \\
\text { dysfunctionb }\end{array}$ & IVMP, Ivlg x2, PoP, RTX ×3 & $\begin{array}{l}41 \text { months. Recovered fully } \\
\text { (mRS 0) }\end{array}$ \\
\hline 2 & $51 / F$ & $\begin{array}{l}N / A^{c} \\
(V G K C \\
\text { positive in } \\
2008)\end{array}$ & $\begin{array}{l}\text { Cognitive impairment, left facial } \\
\text { twitching, and GTC seizure. } \\
\text { Thyroid papillary } \\
\text { microcarcinoma. (mRS 3) }\end{array}$ & $4.2(1.2)$ & $\begin{array}{l}\mathrm{B} / \mathrm{L} \\
\text { mediotemporal } \\
\mathrm{T} 2 \\
\text { hyperintensity }\end{array}$ & $\begin{array}{l}\text { Bitemporal } \\
\text { dysfunction w/ } \\
\text { cortical } \\
\text { irritability }^{\mathrm{b}}\end{array}$ & IVIg, PoP, PLEX, CP X7 & $\begin{array}{l}37 \text { months. Partial recovery, } \\
\text { mildly impaired short-term } \\
\text { memory (mRS 1); St. } \\
\text { postthyreoidectomy }\end{array}$ \\
\hline 3 & $\begin{array}{l}57 / \\
M\end{array}$ & $\begin{array}{l}\text { CSF-LGI1- } \\
\text { Ab } \\
\text { positive }\end{array}$ & $\begin{array}{l}\text { Episodic epigastric sensations w/ } \\
\text { speech difficulty, R FBDS. } \\
\text { Confusion and memory decline } \\
\text { (mRS 4). }\end{array}$ & $4.9(\mathrm{~N} / \mathrm{A})$ & $\begin{array}{l}\mathrm{L} \\
\text { mediotemporal } \\
\text { T2 } \\
\text { hyperintensity }^{\mathrm{d}}\end{array}$ & Normal $^{b}$ & IVMP, RTX x4, PoP & $\begin{array}{l}18 \text { months. Partial recovery; } \\
\text { mild cognitive impairment } \\
\text { (mRS 1) }\end{array}$ \\
\hline 4 & $\begin{array}{l}74 / \\
\mathrm{M}\end{array}$ & $\begin{array}{l}\text { S-LGI1-Ab } \\
\text { positive }\end{array}$ & $\begin{array}{l}\text { Subclinical seizures, confusion, } \\
\text { memory loss, and speech } \\
\text { difficulties (mRS 4). }\end{array}$ & $0.8(\mathrm{~N} / \mathrm{A})$ & $\begin{array}{l}\text { Unrelated } \\
\text { microvascular } \\
\text { changes }\end{array}$ & $\begin{array}{l}\text { Left temporal } \\
\text { epileptic } \\
\text { discharges }^{\text {b }}\end{array}$ & IVMP, PoP, Ivlg x4, RTX x2 & $\begin{array}{l}30 \text { months. Mild residual } \\
\text { behavioral changes (mRS 1) }\end{array}$ \\
\hline 5 & $\begin{array}{l}48 / \\
M\end{array}$ & $\begin{array}{l}\text { S- and } \\
\text { CSF-LGI1- } \\
\text { lgG-Ab } \\
\text { positive }\end{array}$ & $\begin{array}{l}\text { Memory decline, personality } \\
\text { change, GTC, and partial seizures } \\
\text { (mRS 4). }\end{array}$ & $0.6(5.6)$ & $\begin{array}{l}\mathrm{L} \\
\text { mediotemporal } \\
\mathrm{T} 2 \\
\text { hyperintensity }^{\mathrm{d}}\end{array}$ & $\begin{array}{l}\text { Left temporal } \\
\text { epileptic } \\
\text { discharges }^{b}\end{array}$ & IVMP, Ivlg, PoP, RTX ×3 & $\begin{array}{l}25 \text { months. Recovered fully } \\
\text { (mRS 0) }\end{array}$ \\
\hline 6 & $75 / F$ & $\begin{array}{l}\text { S-LGI1-Ab } \\
\text { positive }\end{array}$ & R FBDS, cognitive decline (mRS 3). & $2.3(5.3)$ & Normal & $\begin{array}{l}\text { N/A (performed } \\
\text { abroad } 3 \\
\text { months earlier) }^{\mathrm{b}}\end{array}$ & IVMP, IVlg x7 & $\begin{array}{l}24 \text { months. Partial recovery; } \\
\text { mild cognitive issues (mRS 1). } \\
\text { Invasive mamma-ca operated }\end{array}$ \\
\hline 7 & $\begin{array}{l}63 / \\
M\end{array}$ & $\begin{array}{l}\text { S-LGI1-Ab } \\
\text { positive }\end{array}$ & $\begin{array}{l}\text { L FBDS, SIADH, mild memory } \\
\text { decline (mRS 4). }\end{array}$ & $0.2(11.6)$ & $\begin{array}{l}\text { Unrelated } \\
\text { vascular } \\
\text { compression }\end{array}$ & $\begin{array}{l}\text { Right temporal } \\
\text { epileptic } \\
\text { discharges }^{\text {b }}\end{array}$ & $\begin{array}{l}\text { IVMPe } \times 3, \text { PoP, PLEX, RTX } \\
\text { x3 }\end{array}$ & $\begin{array}{l}12 \text { months. Relapsed x2, } \\
\text { improved (mRS } 1) \text {, lost to } \\
\text { further follow-up }\end{array}$ \\
\hline 8 & $\begin{array}{l}57 / \\
M\end{array}$ & $\begin{array}{l}\text { S-LGI1-Ab } \\
\text { positive }\end{array}$ & $\begin{array}{l}\text { R FBDS, GTC seizure, episodic } \\
\text { confusion, abulia, fasciculations, } \\
\text { and ataxia (mRS 4). }\end{array}$ & $3.2(0.5)$ & $\begin{array}{l}\text { Unrelated } \\
\text { microvascular } \\
\text { changes }\end{array}$ & Normal & IVMP, Ivlg ×5, RTX ×3, PoP & $\begin{array}{l}20 \text { months. Recovered fully } \\
\text { (mRS 0) }\end{array}$ \\
\hline 9 & $\begin{array}{l}65 / \\
M\end{array}$ & $\begin{array}{l}\text { S-LGI1-Ab } \\
\text { positive }\end{array}$ & $\begin{array}{l}\text { Rapidly progressive dementia, } \\
\text { hyponatremia, and L FBDS } \\
\text { (mRS 4). }\end{array}$ & $8.1(N / A)$ & $\begin{array}{l}\text { L } \\
\text { mediotemporal } \\
\text { T2 } \\
\text { hyperintensity } \\
\text { w/Gd+ }\end{array}$ & $\begin{array}{l}\text { Mild } L>R \\
\text { cerebral } \\
\text { dysfunction }^{b}\end{array}$ & IVlge, IVMPe, PoP, RTX x1 & $\begin{array}{l}24 \text { months. Partial recovery; } \\
\text { mild to moderate memory } \\
\text { impairment, and requires } \\
\text { some help (mRS 3) }\end{array}$ \\
\hline 10 & $\begin{array}{l}78 / \\
M\end{array}$ & $\begin{array}{l}\text { S-LGI1- } \\
\text { IgG-Ab } \\
\text { positive }\end{array}$ & $\begin{array}{l}\text { Right FBDS, mild memory decline } \\
\text { (mRS 1). }\end{array}$ & $5.4(\mathrm{~N} / \mathrm{A})$ & $\begin{array}{l}\text { L putaminal T2 } \\
\text { hyperintensity } \\
\text { w/diffusion } \\
\text { restriction }\end{array}$ & Normal & IVMPe $\times 9$, PoP & $\begin{array}{l}21 \mathrm{~d} \text {. Improved; memory } \\
\text { impairment (mRS 1). Lost to } \\
\text { further follow-up }\end{array}$ \\
\hline 11 & $65 / F$ & $\begin{array}{l}\text { S- and } \\
\text { CSF-LGI1- } \\
\text { lgG-Ab } \\
\text { positive }\end{array}$ & $\begin{array}{l}\text { L FBDS, atonic seizures, memory } \\
\text { decline (mRS 4). }\end{array}$ & $1.0(\mathrm{~N} / \mathrm{A})$ & $\begin{array}{l}\text { R medio- } \\
\text { temporal Gd+ }\end{array}$ & $\begin{array}{l}\text { Bitemporal } \\
\text { epileptic } \\
\text { discharges }\end{array}$ & IvMPe, Ivlge, PoP, RTX x 2 & $\begin{array}{l}13 \text { months. Recovered fully }{ }^{f} \\
\text { (mRS 0) }\end{array}$ \\
\hline 12 & $70 / F$ & $\begin{array}{l}\text { S- and } \\
\text { CSF-LGI1- } \\
\text { lgG-Ab } \\
\text { positive }\end{array}$ & $\begin{array}{l}\text { B/L FBDS, cognitive decline, } \\
\text { seizures, hyponatremia, } \\
\text { choreiform movements, and } \\
\text { impaired balance (mRS 5). }\end{array}$ & $13.7(5.8)$ & $\begin{array}{l}\text { B/L restricted } \\
\text { cortical } \\
\text { diffusion, } \\
\text { cerebellar Gd+ }\end{array}$ & $\begin{array}{l}\text { Left } \\
\text { frontotemporal } \\
\text { dysfunction }\end{array}$ & IVMP, PoP, RTX x2 & $\begin{array}{l}13 \text { months. Recurring disease } \\
\text { activity. Improved; memory } \\
\text { impairment, impulsivity, and } \\
\text { in assisted living ( } \mathrm{mRS} 3 \text { ) }\end{array}$ \\
\hline 13 & $70 / F$ & $\begin{array}{l}\text { S-LGI1- } \\
\text { IgG-Ab } \\
\text { positive }\end{array}$ & $\begin{array}{l}\text { Cognitive decline, short-term } \\
\text { memory loss, auditory } \\
\text { hallucinations, and } \\
\text { hyponatremia (mRS 4). }\end{array}$ & $8.4(7.0)$ & $\begin{array}{l}\text { B/L } \\
\text { mediotemporal } \\
\text { T2 } \\
\text { hyperintensity }\end{array}$ & Normal & IVMP, RTXx 5 & $\begin{array}{l}19 \text { months. Improved; mild } \\
\text { forgetfulness and impulsivity, } \\
\text { and anger issues (mRS 1) }\end{array}$ \\
\hline
\end{tabular}

Abbreviations: $a 3-A C h R=$ ganglionic alpha 3-acetylcholine receptor; $\mathrm{Ab}=$ antibody; $\mathrm{AE}=$ autoimmune encephalitis, $\mathrm{APS}=$ antiphospholipid syndrome; $\mathrm{B} / \mathrm{L}=$ bilateral; $C P=$ cyclophosphamide; D-SM-PNP = demyelinating sensorimotor polyneuropathy; FDG $=\left[{ }^{18} \mathrm{~F}\right]$ fluorodeoxyglucose; FBDS = facio-brachial dystonic seizure; Gd+ = Gadolinium enhancement; GTC = generalized tonic-clonic; HE = Hashimoto encephalopathy; IVMP = IV methylprednisolone (1 g daily for 3-5 days); Ivlg = IV Immunoglobulin (doses ranging from 1.0 to $2.0 \mathrm{~g} / \mathrm{kg}$ over 2-5 days); L = left; LGI1 = Leucine-rich, glioma-inactivated-1; mRS = modified Rankin scale; PLEX = plasma exchange (5 cycles); $\mathrm{PoP}=$ peroral prednisone, tapered; $\mathrm{R}=$ right; $\mathrm{RTX}=\mathrm{IV}$ rituximab $(1,000$ mg infusion); $\mathrm{S}=$ serum; $\mathrm{VGKC}=\mathrm{voltage}$-gated potassium channel.

N.B. The Number of Treatment Cycles (for IVMP or Ivlg) or infusions (for RTX) marked, for example, x2.

a Follow-up time reported as the time from the first $\left[{ }^{18} \mathrm{~F}\right] \mathrm{FDG}$ PET scan to the latest available neurologic follow-up.

${ }^{b}$ Antiepileptic medication in use at the time of the first $\left[{ }^{18} \mathrm{~F}\right] \mathrm{FDG}$ PET scan.

'The patient had a positive S-VGKC titer $(1.03 \mathrm{nmol} / \mathrm{L})$, and the initial diagnosis and treatment were pursued in $2008 \mathrm{before}$ the description of LGI 1 as target antigen.

${ }^{\mathrm{d}}$ An MRI performed before this baseline MRI had been normal.

e The marked immunosuppressive medication already given or initiated the time of the first $\left[{ }^{18} \mathrm{~F}\right] \mathrm{FDG}$ PET scan.

${ }^{\mathrm{f}}$ Neurology follow-up ongoing. 
Table 2 Demographic, Clinical, Antibody Testing, MRI, and EEG Characteristics of the Non-LGI1-AE Group

\begin{tabular}{|c|c|c|c|c|c|c|c|c|}
\hline $\begin{array}{l}\text { Case } \\
\text { no }\end{array}$ & $\begin{array}{l}\text { Age } \\
\text { (y)/sex }\end{array}$ & $\begin{array}{l}\text { Diagnosis, } \\
\text { antibody test } \\
\text { result }\end{array}$ & $\begin{array}{l}\text { Clinical } \\
\text { presentation } \\
\text { before the first PET }\end{array}$ & $\begin{array}{l}\text { Time from } \\
\text { onset to first } \\
\text { PET (mo) }\end{array}$ & $\begin{array}{l}\text { MRI at the time } \\
\text { of first PET }\end{array}$ & $\begin{array}{l}\text { EEG at the time } \\
\text { of first PET }\end{array}$ & $\begin{array}{l}\text { Immunomodulatory } \\
\text { treatments }\end{array}$ & $\begin{array}{l}\text { Neurology follow-up } \\
\text { time. }{ }^{\text {a Clinical outcome, }} \\
\text { other notable }\end{array}$ \\
\hline 14 & $18 / F$ & $\begin{array}{l}\text { NMDA-r AE } \\
\text { CSF- and S-NR1/ } \\
\text { NR2-Ab positive }\end{array}$ & $\begin{array}{l}\text { Psychosis, catatonia, } \\
\text { fever, GTC and focal } \\
\text { seizures }\end{array}$ & 0.5 & $\begin{array}{l}\text { Unrelated nonspecific } \\
\text { subcortical white } \\
\text { matter changes }\end{array}$ & $\begin{array}{l}\text { Generalized } \\
\text { slowing }\end{array}$ & $\begin{array}{l}\text { Salpingo-oophorectomy, } \\
\text { PLEX x5, Ivlg x1 }\end{array}$ & $\begin{array}{l}11 \text { months. Near full } \\
\text { recovery; mild decline in } \\
\text { neuropsychologic testing } \\
\text { f/expected baseline }\end{array}$ \\
\hline 15 & $24 / F$ & $\begin{array}{l}\text { NMDA-r AE } \\
\text { CSF- and S-NR1-Ab } \\
\text { positive }\end{array}$ & $\begin{array}{l}\text { Psychosis, } \\
\text { dysautonomia, oral } \\
\text { dyskinesia/ } \\
\text { automatism, and } \\
\text { malignant catatonia }\end{array}$ & 0.9 & $\begin{array}{l}\text { B/L mediotemporal } \\
\text { and subinsular } \\
\text { hyperintensity in } \\
\text { FLAIR }\end{array}$ & $\begin{array}{l}\text { Diffuse } \\
\text { slowing }\end{array}$ & $\begin{array}{l}\text { IVMP x1, IVIg x1, B/L } \\
\text { oophorectomy }\end{array}$ & $\begin{array}{l}2 \text { months. Near full } \\
\text { recovery; residual anxiety }\end{array}$ \\
\hline 16 & $62 / F$ & $\begin{array}{l}\text { NMDA-r AE } \\
\text { CSF- and S-NMDA-r } \\
\text { Ab positive }\end{array}$ & $\begin{array}{l}\text { Headache and } \\
\text { short-term } \\
\text { memory deficit }\end{array}$ & 2.0 & $\begin{array}{l}\text { B/L mediotemporal } \\
(L>R) T 2- \\
\text { hyperintensity } \\
\text { w/leptomeningeal Gd+ }\end{array}$ & $\begin{array}{l}\text { Bitemporal } \\
\text { L>R slowing }\end{array}$ & IVMP x1 & $\begin{array}{l}17 \mathrm{~d} \text {. Partial recovery; daily } \\
\text { headaches and some } \\
\text { memory deficits }\end{array}$ \\
\hline 17 & $70 / \mathrm{M}$ & $\begin{array}{l}\text { NMDA-r AE } \\
\text { CSF-NMDA-r-Ab } \\
\text { positive }\end{array}$ & $\begin{array}{l}\text { LUE jerking and } \\
\text { clumsiness, weight } \\
\text { loss, subacute } \\
\text { memory impairment, } \\
\text { and fatigue }\end{array}$ & 3.2 & $\begin{array}{l}\mathrm{R} \text { basal ganglia, } \mathrm{R} \\
\text { mediotemporal, and } \\
\text { B/L hippocampal } \\
\text { Gd+. Central sulcus } \\
\text { FLAIR hyperintensity } \\
\text { w/leptomeningeal } \\
\text { Gd+. }\end{array}$ & Normal $^{b}$ & $\begin{array}{l}\operatorname{lvlg}^{c}, \text { IVMP, PoP, } \\
\text { Ivlg } \times 6\end{array}$ & $\begin{array}{l}8 \text { months. Near full } \\
\text { recovery; no symptoms } \\
\text { while still on PoP at follow- } \\
\text { up }\end{array}$ \\
\hline 18 & $59 / F$ & $\begin{array}{l}\text { SS-associated AE } \\
\text { S-Ro-Ab } 190 \text { EU/ } \\
m L, S-L a-A b A B 119 \\
\text { EU/mL }\end{array}$ & $\begin{array}{l}\text { Seizures, memory } \\
\text { loss }\end{array}$ & 0.4 & $\begin{array}{l}\mathrm{B} / \mathrm{L} \text { mesial temporal } \\
\mathrm{T} 2 \text { hyperintensity }\end{array}$ & $\begin{array}{l}\text { Numerous } \\
\text { bitemporal } \\
\text { discharges }^{\mathrm{b}}\end{array}$ & IVMP & $\begin{array}{l}27 \text { months. Partial } \\
\text { recovery; persistent short- } \\
\text { term memory impairment }\end{array}$ \\
\hline 19 & $74 / F$ & $\begin{array}{l}\mathrm{HE} \\
\text { S-TPO-Ab } \\
787 \mathrm{IU} / \mathrm{mL}\end{array}$ & $\begin{array}{l}\text { Depression with } \\
\text { psychotic features, } \\
\text { anxiety, and } \\
\text { dementia }\end{array}$ & 11.8 & Normal & B/L slowing & IVMP x1, PoP & $\begin{array}{l}10 \text { months. Mild } \\
\text { improvement; remaining } \\
\text { memory decline and } \\
\text { impulsivity }\end{array}$ \\
\hline 20 & $62 / F$ & $\begin{array}{l}\mathrm{HE} \\
\text { S-TPO-Ab } \\
339.6 \mathrm{IU} / \mathrm{mL}\end{array}$ & $\begin{array}{l}\text { Confusion, headache, } \\
\text { expressive aphasia, } \\
\text { and GTC seizures }\end{array}$ & 3.5 & $\begin{array}{l}\text { L posterior temporal } \\
\text { WM FLAIR } \\
\text { abnormality w/low } \\
\text { diffusivity and Gd+ }\end{array}$ & $\begin{array}{l}\text { L temporal epileptic } \\
\text { discharges }^{\mathrm{b}}\end{array}$ & IVMP x1, PoP & $\begin{array}{l}49 \text { months. Partial } \\
\text { recovery: mild cognitive } \\
\text { impairment, suspicion of } \\
\text { underlying } \\
\text { neurodegenerative } \\
\text { disorder but no } \\
\text { progression }\end{array}$ \\
\hline 21 & $68 / F$ & $\begin{array}{l}\mathrm{HE} \\
S-T G-A b \\
353.51 \mathrm{U} / \mathrm{mL}\end{array}$ & $\begin{array}{l}\text { Seizures, nonfluent } \\
\text { expressive aphasia, } \\
\text { hyperreflexia, ocular } \\
\text { clonus; and severe } \\
\text { pneumonia }\end{array}$ & 13.0 & Normal & $\begin{array}{l}\text { Multifocal } \\
\text { epileptic } \\
\text { discharges }^{b}\end{array}$ & $\begin{array}{l}\text { IVMP x1, PoP, } \\
\text { IVMP x1, IVIg x1 }\end{array}$ & $\begin{array}{l}34 \text { months. Relapse } \\
\text { w/paranoia, visual } \\
\text { hallucinations. Partial } \\
\text { recovery; and mild } \\
\text { cognitive impairment }\end{array}$ \\
\hline 22 & $63 / F$ & $\begin{array}{l}\text { Anti-Zic4/GAD65 } \\
\text { AE } \\
\text { S-Zic4-Ab and S- } \\
\text { GAD-65-Ab positive }\end{array}$ & $\begin{array}{l}\text { Ataxia, tremor, } \\
\text { diplopia, dysarthria; } \\
\text { and small cell lung } \\
\text { cancer }\end{array}$ & 9.6 & $\begin{array}{l}\text { Numerous unrelated } \\
\text { nonspecific WM } \\
\text { changes }\end{array}$ & N/A & $\begin{array}{l}\text { Ivlg } \times 1 \text { (+dexamethasone, } \\
\text { carboplatin, and } \\
\text { etoposide) }\end{array}$ & $\begin{array}{l}5 \mathrm{~d} \text {. Stable but persisting } \\
\text { neurologic symptoms with } \\
\text { no improvement }\end{array}$ \\
\hline 23 & $64 / M$ & $\begin{array}{l}\text { Anti-GAD-65 AE } \\
\text { S-GAD-Ab } \\
0.23 \mathrm{nmol} / \mathrm{L}\end{array}$ & $\begin{array}{l}\text { Opsoclonus- } \\
\text { myoclonus, limb } \\
\text { ataxia and dysmetria, } \\
\text { upbeat nystagmus, } \\
\text { dysphagia; PE, } \\
\text { suspicion of lung } \\
\text { malignancy }\end{array}$ & 4.2 & $\begin{array}{l}\text { Unrelated ischemic } \\
\text { WM changes }\end{array}$ & $N / A$ & $\begin{array}{l}\text { IvMP x1, mycophenolate, } \\
\text { PoP, IVMP x1, PoP }\end{array}$ & $\begin{array}{l}5 \text { months. Partial initial } \\
\text { recovery. New episode } \\
\text { w/worsening symptoms } \\
\text { and delirium; remaining } \\
\text { opsoclonus, mild delirium, } \\
\text { mild dysarthria, and } \\
\text { dysmetria }\end{array}$ \\
\hline 24 & $66 / F$ & $\begin{array}{l}\text { Anti-Hu AE } \\
\text { S-Hu-Ab positive }\end{array}$ & $\begin{array}{l}\text { Seizures, lethargy, } \\
\text { confusion, reduced } \\
\text { consciousness; and } \\
\text { small cell lung cancer }\end{array}$ & 2.3 & $\begin{array}{l}\text { Mild L mediotemporal } \\
\text { hyperintensity }\end{array}$ & $\begin{array}{l}\text { Multifocal L > R } \\
\text { epileptic } \\
\text { discharge }^{b}\end{array}$ & Ivlg x1, IVMP x1, PoP & $\begin{array}{l}15 \mathrm{~d} \text {. Mild improvement, } \\
\text { but later progressed; and } \\
\text { deceased after latest } \\
\text { neurology follow-up }\end{array}$ \\
\hline 25 & $77 / M$ & $\begin{array}{l}\text { APS-associated } \\
\text { AE } \\
\text { S-Beta-2- } \\
\text { glycoprotein I } \\
\text { Ab }>150 \mathrm{U} / \mathrm{mL}^{\mathrm{d}}\end{array}$ & $\begin{array}{l}\text { Progressive cognitive } \\
\text { decline, mood } \\
\text { changes, chorea and } \\
\text { mild tremor, } \\
\text { dysphagia, } \\
\text { oromandibular } \\
\text { dyskinesia; and } \\
\text { multiple previous } \\
\text { strokes }\end{array}$ & 36.6 & $\begin{array}{l}\text { Multiple } \\
\text { microvascular WM } \\
\text { changes; old infarcts } \\
\text { in cerebellum, R } \\
\text { occipital lobe, R } \\
\text { centrum semiovale, } \\
\text { and L thalamus }\end{array}$ & $\mathrm{N} / \mathrm{A}$ & $\begin{array}{l}\text { No immunomodulatory } \\
\text { treatment (varfarine } \\
\text { initiated) }\end{array}$ & $\begin{array}{l}15 \text { months. Partial } \\
\text { recovery at latest } \\
\text { neurology follow-up; } \\
\text { deceased later due to } \\
\text { acute metabolic } \\
\text { encephalopathy and } \\
\text { aspiration pneumonia }\end{array}$ \\
\hline 26 & $70 / F$ & $\begin{array}{l}\text { Anti-GAD-65 and } \\
\text { D-SM- } \\
\text { PNP-associated } \\
\text { AE } \\
\text { S-GAD-65 and } \\
\text { S-a3-AChR-Ab } \\
\text { positive }\end{array}$ & $\begin{array}{l}\text { Confusion and } \\
\text { progressive } \\
\text { weakness of upper } \\
\text { and lower limbs }\end{array}$ & 0.9 & $\begin{array}{l}\text { Unrelated old L } \\
\text { frontal ischemic } \\
\text { infarct and unrelated } \\
\text { microvascular WM } \\
\text { changes }\end{array}$ & Bilateral slowing & $\operatorname{lvlg} \times 1$ & $\begin{array}{l}3 \text { months. Full cognitive } \\
\text { recovery, peripheral } \\
\text { neuropathic pain } \\
\text { remained }\end{array}$ \\
\hline
\end{tabular}


Table 2 Demographic, Clinical, Antibody Testing, MRI, and EEG Characteristics of the Non-LGI1-AE Group (continued)

\begin{tabular}{|c|c|c|c|c|c|c|c|c|}
\hline $\begin{array}{l}\text { Case } \\
\text { no }\end{array}$ & $\begin{array}{l}\text { Age } \\
\text { (y)/sex }\end{array}$ & $\begin{array}{l}\text { Diagnosis, } \\
\text { antibody test } \\
\text { result }\end{array}$ & $\begin{array}{l}\text { Clinical } \\
\text { presentation } \\
\text { before the first PET }\end{array}$ & $\begin{array}{l}\text { Time from } \\
\text { onset to first } \\
\text { PET (mo) }\end{array}$ & $\begin{array}{l}\text { MRI at the time } \\
\text { of first PET }\end{array}$ & $\begin{array}{l}\text { EEG at the time } \\
\text { of first PET }\end{array}$ & $\begin{array}{l}\text { Immunomodulatory } \\
\text { treatments }\end{array}$ & $\begin{array}{l}\text { Neurology follow-up } \\
\text { time. }{ }^{\text {a Clinical outcome, }} \\
\text { other notable }\end{array}$ \\
\hline 27 & $55 / \mathrm{M}$ & $\begin{array}{l}\text { CASPR2 AE } \\
\text { S-CASPR2-Ab } \\
\text { positive }\end{array}$ & $\begin{array}{l}\text { Cognitive decline, } \\
\text { hallucinations, } \\
\text { fasciculations, } \\
\text { copious salivation, } \\
\text { and malaise }\end{array}$ & 2.5 & $\begin{array}{l}\text { Unrelated } \\
\text { microvascular } \\
\text { changes }\end{array}$ & $\begin{array}{l}\text { Nonspecific } \\
\text { slowing }\end{array}$ & $\begin{array}{l}\text { IVMP x2, PLEX, RTX x2, } \\
\text { IVlg, PoP. Thymectomy } \\
\text { for thymoma. }\end{array}$ & $\begin{array}{l}6 \text { months. Partial } \\
\text { recovery; mild cognitive } \\
\text { issues, and slightly altered } \\
\text { personality }\end{array}$ \\
\hline 28 & $72 / M$ & $\begin{array}{l}\text { GABA-B-r AE } \\
\text { CSF- and S-GABA-B- } \\
r-A b \text { positive }\end{array}$ & $\begin{array}{l}\text { GTC seizure, } \\
\text { agitation, and } \\
\text { confusion }\end{array}$ & 4.9 & $\begin{array}{l}\text { Nonrelated general } \\
\text { atrophy, WM } \\
\text { changes, and a } \\
\text { cerebellar AVM }\end{array}$ & $\begin{array}{l}\text { L temporal cortical } \\
\text { irritability, } L>R \\
\text { bitemporal } \\
\text { dysfunction }^{\text {b }}\end{array}$ & $\begin{array}{l}\text { IVlgc }^{c}, I^{\prime V M} P^{c}, P^{\prime} X^{c} \\
\text { PoP }^{c}, \text { RTX }\end{array}$ & $\begin{array}{l}\text { Lost to neurology follow- } \\
\text { up. Partial initial recovery, } \\
\text { and no long-term } \\
\text { outcome data available }\end{array}$ \\
\hline
\end{tabular}

Abbreviations: $a 3-A C h R=$ ganglionic alpha3-acetylcholine receptor; $A b=$ antibody; $A E=$ autoimmune encephalitis, $A P S=$ antiphospholipid syndrome; $B / L=$ bilateral; D-SM-PNP = demyelinating sensorimotor polyneuropathy; FDG = ${ }^{18}$ F]fluorodeoxyglucose; Gd+ = Gadolinium enhancement; GTC = generalized tonic-clonic; $\mathrm{HE}=$ Hashimoto encephalopathy; IVMP = IV methylprednisolone (1 g daily for 3-5 days); IVlg = IV Immunoglobulin (doses ranging from 1.0 to 2.0 g/kg over 2-5 days); L = left; LGI1 = Leucine-rich, glioma-inactivated-1; LUE = left upper extremity; PE = pulmonary embolism; PLEX = plasma exchange (5 cycles); PoP = peroral prednisone, tapered; R = right; RTX = IV rituximab (1,000 mg infusion); S = serum; SS = Sjögren syndrome; TG = thyreoglobulin; TPO = thyroid peroxidase; VGKC = voltage-gated potassium channel.

N.B. The number of treatment cycles (for IVMP or Ivlg) or infusions (for RTX) marked, for example, $x 2$.

${ }^{a}$ Follow-up time reported as the time from the first $\left[{ }^{18} \mathrm{~F}\right]$ FDG PET scan to the latest available neurologic follow-up.

${ }^{\mathrm{b}}$ Antiepileptic medication in use at the time of the first $\left[{ }^{18} \mathrm{~F}\right]$ FDG PET scan.

${ }^{c}$ The marked immunosuppressive medication already given or initiated at the time of the first $\left[{ }^{18} \mathrm{~F}\right] \mathrm{FDG}$ PET scan

${ }^{d}$ The patient was also examined for LGI1 and other neuronal antibodies. The CSF Encephalopathy Autoimmune Panel (including CSF-VGKC-AB and CSF-LGI1$A B$ ) and Serum Paraneoplastic antibody evaluation (including neuronal VGKC-AB) showed negative results.

the LGI1-AE group vs non-LGI1-AE group and LGI1-AE group vs $\mathrm{AD}$ group comparisons. Finally, $\mathrm{P} / \mathrm{Mi}$ was the only index providing values of $\mathrm{LR}+>10$ and $\mathrm{LR}-<0.20$ in all group comparisons (eTable 4, links.lww.com/NXI/A695). As defined by the $\mathrm{P} / \mathrm{Mi}$ cutoff value, there was one falsepositive case in the non-LGI1-AE group who was a patient with APS-associated AE (Figure 1C) with a P/Mi-value of 1.57 .

\section{MTL Hypermetabolism Is Associated With Concurrent Disability in LGI1-AE}

LGI1-AE patients with moderately severe-to-severe disability $(\mathrm{mRS}>3)$ showed higher MTL-SUVRg when compared with those with mild-to-moderate disability $(\mathrm{mRS} \leq 3)$ at baseline $(+14 \%, p=0.028)$ (Figure 2B). However, no difference was observed in MTL-SUVRg between patients with normal vs abnormal EEG findings at the time of PET (median [IQR] 0.83 [0.20] vs 0.91 [0.07], $p=0.368$ ). On the other hand, no difference was observed in P-SUVRg in LGI1-AE patients with $\mathrm{mRS}>3$ when compared with those with $\mathrm{mRS} \leq 3$ $(+6.2 \%, p=0.287$; Figure 2B).

\section{Dysmetabolism Extends Beyond Putamen and Mediotemporal Lobe in LGI1-AE}

There was widespread hypometabolism in several, largely neocortical regions in LGI1-AE when compared with NCs. Specifically, on ROI analysis, cortical SUVRg values were lower in several frontal lobe regions including opercular, triangular, and orbital parts of inferior frontal gyrus; medial orbital part of superior frontal gyrus and gyrus rectus; and in several parietal lobe regions including angular gyrus and inferior parietal lobe $(-4.7$ to $-7.2 \%$, all $p<0.05$ ). Conversely, olfactory and fusiform gyri and occipital inferior lobe showed hypermetabolism in LGI1-AE group when compared with NCs $(+5.9$ to $+11.4 \%$, all $p<0.05)$ (Figure 3A, eTable 5, links.lww.com/NXI/A695).
Several additional subcortical structures, including caudate, pallidum, and pons, demonstrated hypermetabolism in LGI1$\mathrm{AE}$ group when compared with $\mathrm{NCs}(+7.3$ to +12.5 , all $p<0.05$; Figure 3B, eTable 5, links.lww.com/NXI/A695). None of these additional cortical or subcortical structures showed associations between baseline mRS and their respective SUVRg values.

\section{Metabolic Changes in LGI1-AE After Immunosuppressive Treatment and Their Relationship With Short-Term Outcomes}

All patients with LGI1-AE received immunosuppressive treatment, and short-term follow-up FDG-PET images were available for 8 patients with LGI1-AE (Table 1). The mRS scores reduced from baseline to short-term follow-up (from median [IQR] mRS 4 [2] to $2[2] ; p=0.008$ ) (eFigure 1 , links.lww.com/NXI/A695).

\section{Normalization of Mediotemporal Hypermetabolism After Treatment}

MTL-SUVRg decreased on follow-up in LGI1-AE (-11.3\%, $p=0.050$, significant at $p=0.050)$ and was no longer higher than that in NCs at follow-up $(p=0.829)$ (Figure 4A, eTable 6, links.lww.com/NXI/A695).

Of importance, patients with good short-term outcome (mRS $\leq 2$ ) at the time of follow-up PET showed a reduction in MTL hypermetabolism $(-19.8 \%, p=0.043)$, as opposed to those with a poorer short-term outcome $(\mathrm{mRS}>2)$, who did not show changes $(+6.0 \%, p=1.000)$ when compared with their baseline. Furthermore, on comparing patients with $\mathrm{mRS}>2$ with patients with $\mathrm{mRS} \leq 2$, the former demonstrated higher follow-up SUVRg values (effect size $-0.79, p=0.036$ ) at the time of follow-up PET (eFigure 2, eTable 7, links.lww.com/ NXI/A695). Individual changes in mediotemporal metabolism are visualized for case 5 in Figure 4B. Changes in MTL/Th and 
Figure 1 Regional [18F]FDG-PET Findings in LGI1- and Non-LGI1 AE Groups, Alzheimer Disease Group, and NCs
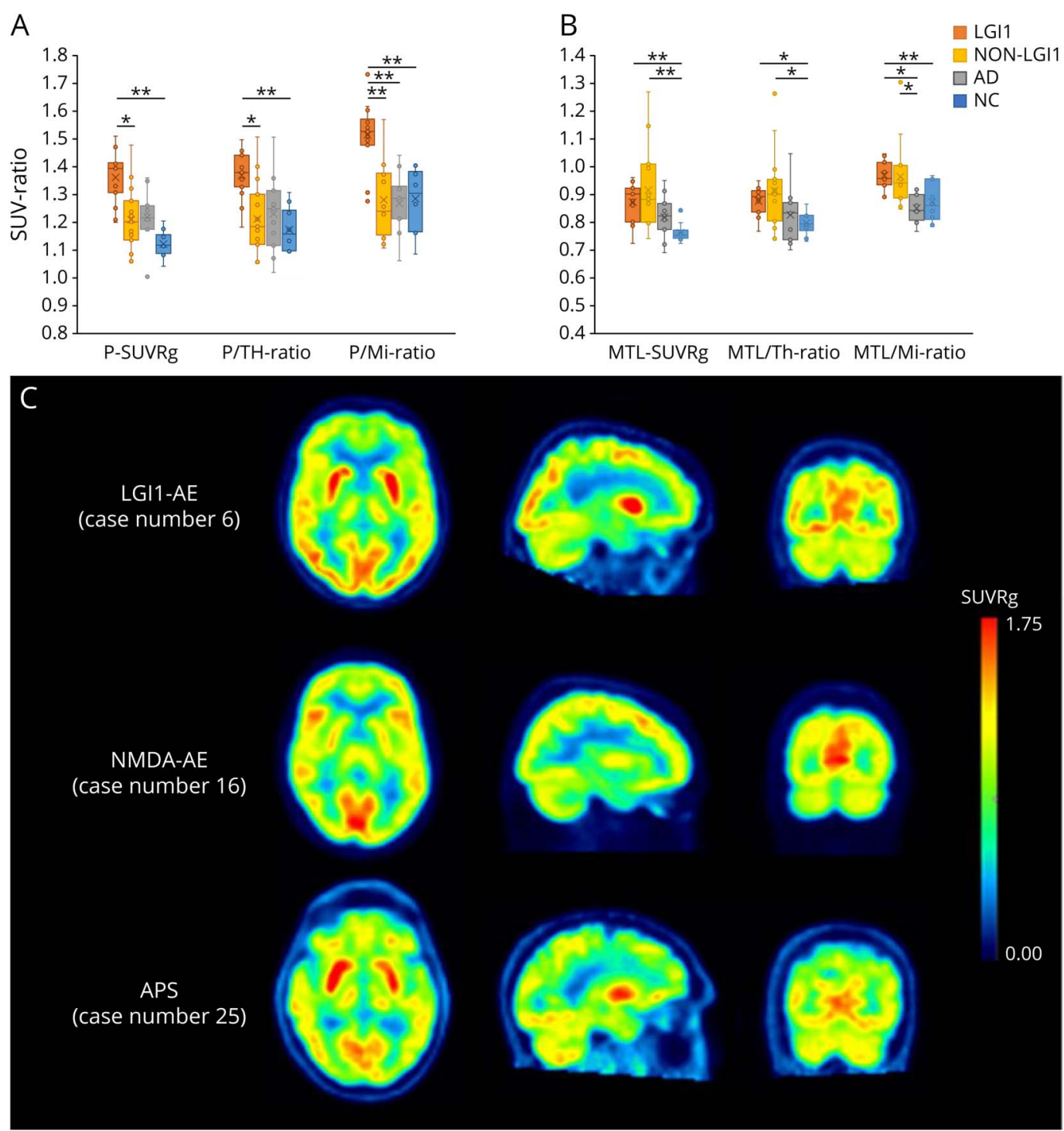

Metabolic activity in (A) putamen and (B) MTL, measured as standardized uptake value ratios (SUVR) normalized to global brain, thalamus and midbrain. (C) Visualization of metabolic abnormalities in individual patients in LGI1-AE and non-LGI1-AE groups using parametric, global normalized [ $\left.{ }^{18} \mathrm{~F}\right] \mathrm{FDG}$ SUVR images. The images represent patients with LGI1-AE (case 6), NMDAr (case 16), and APS-associated (case 25) autoimmune encephalitides (AE) and demonstrate putaminal hypermetabolism and relative occipital hypermetabolism in LGI1-AE, lateral occipital hypometabolism and normal putaminal metabolism in NMDAr-AE, and pronounced putaminal hypermetabolism in APS-AE. Numbering of the patient cases refers to the case numbers in Tables 1 and $2 .{ }^{*} p<0.05$ and ${ }^{*} p<0.01$ (significant at the level of $\left.\left.p<0.05\right) .{ }^{18} \mathrm{~F}\right] \mathrm{FDG}=\left[{ }^{18} \mathrm{~F}\right]$ fluorodeoxyglucose; $\mathrm{AD}=$ Alzheimer disease; $\mathrm{AE}=$ autoimmune encephalitis; APS = antiphospholipid syndrome; LGI1 = leucine-rich, glioma-inactivated-1; Mi = midbrain; MTL = mediotemporal lobe; $\mathrm{NC}=$ negative control; Non-LGI1 = non-LGI1 autoimmune encephalitis; $\mathrm{P}$ = putamen; SUV = standardized uptake value; SUVRg = global brain normalized standardized uptake value ratio; $\mathrm{Th}=$ thalamus.

MTL/Mi values after immunotherapy are depicted in Figure 4A and summarized in eTable 6, links.lww.com/NXI/A695.

\section{Changes in Putaminal Hypermetabolism After Treatment}

Significant reduction in putaminal uptake at follow-up was observed for SUVRg in LGI1-AE group $(-11.2 \%$; $p<0.012)$, but it remained higher than that in NCs $(+10.1 \%, p=0.013)$ (Figure 4A, eTable 6, links.lww.com/NXI/A695). Of interest, when stratified by short-term clinical outcome, P-SUVRg reduced from baseline to follow-up in the $\mathrm{mRS} \leq 2$ group $(-12.5 \%, p=0.043)$ but not in the mRS $>2$ group $(-9.9 \%, p=$ $0.109)$. However, short-term follow-up PET did not demonstrate differences in putaminal metabolism between the good and poor short-term outcome groups (effect size -0.26, $p=$ 0.036) (eFigure 2, eTable 7, links.lww.com/NXI/A695). Individual putaminal metabolic changes are demonstrated in case 6 in Figure 4C. Changes in $\mathrm{P} / \mathrm{Th}$ and $\mathrm{P} / \mathrm{Mi}$ after immunosuppressive treatment are shown in Figure 4A and summarized in eTable 6, links.lww.com/NXI/A695. 
Figure 2 Diagnostic Accuracy of Putaminal-[18F]FDG Indices and Relationship of Mediotemporal and Putaminal Dysmetabolism With mRS in LGI1-AE

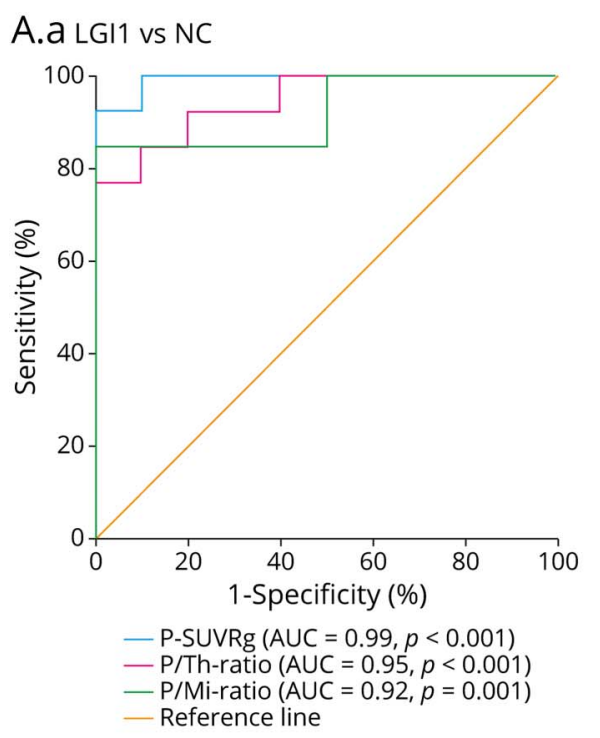

B

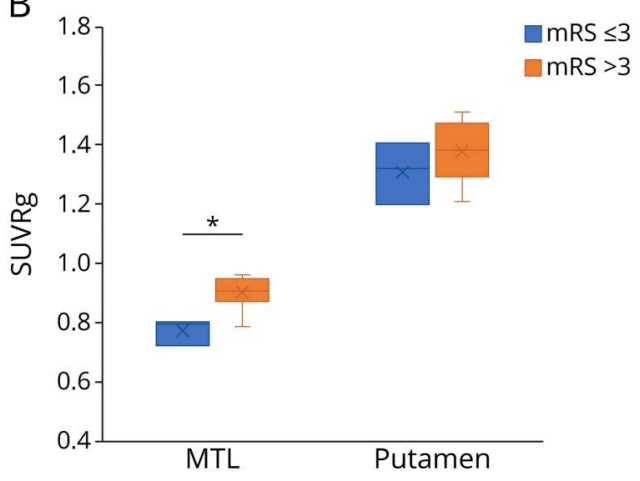

A.b LGI1 vs Non-LGl1

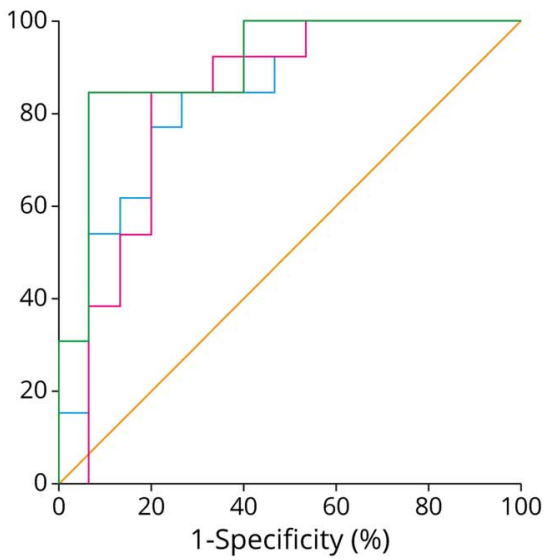

- P-SUVRg (AUC $=0.84, p=0.003$ )

- P/Th-ratio (AUC $=0.83, p=0.003$ )

- P/Mi-ratio (AUC $=0.90, p<0.001)$

- Reference line
A.C LGI1 vs AD

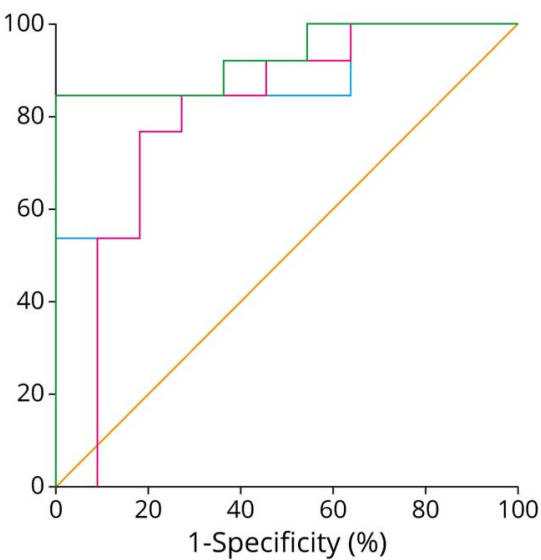

- P-SUVRg (AUC $=0.84, p=0.005$ )

- P/Th-ratio $(A \cup C=0.80, p=0.012)$

- P/Mi-ratio (AUC $=0.93, p<0.001)$

- Reference line

(A.a-c) Receiver operating characteristic (ROC) curve analyses of $\left[{ }^{18} \mathrm{~F}\right] \mathrm{FDG}$ putaminal indices for the evaluation of their accuracy in differentiating patients with LGI1-AE from NCs (A.a), non-LGI1-AE group (A.b) and AD group (A.c). All $p$ values are significant at the level of $p<0.05$. (B) Comparison of mediotemporal and putaminal metabolic activity, measured as global brain normalized standardized uptake value ratios (SUVRg) of [ ${ }^{18} \mathrm{~F}$ ]FDG, in LGI1-AE group with mild-tomoderate disability (mRS $\leq 3)$ vs moderately severe-to-severe disability $\left(\mathrm{mRS}>3\right.$ ) at the time of the initial $\left.{ }^{18} \mathrm{~F}\right] \mathrm{FDG}$-PET. * $p<0.05$ (significant at the level of $p<$ 0.05). $\left[{ }^{18} \mathrm{~F}\right] \mathrm{FDG}=\left[{ }^{18} \mathrm{~F}\right]$ fluorodeoxyglucose; $A D=$ Alzheimer disease; $A E=$ autoimmune encephalitis; $A U C=$ area under the curve; $L G I 1=$ leucine-rich, glioma-inactivated-1; Mi = midbrain; mRS = modified Rankin scale; $M$ TL = mediotemporal lobe; NCs = negative controls; non-LGI1 = non-LGI1 autoimmune encephalitis; P = putamen; SUVRg = global brain normalized SUV ratio; Th = thalamus.

\section{Changes in Metabolism in Neocortical and Other Gray Matter Regions}

Significant increases were observed in cortical metabolism after treatment and were associated with concurrent shortterm clinical improvement. Specifically, cortical SUVRg values increased in frontal (middle and opercular part of inferior frontal), occipital (posterior cingulum, calcarine, cuneus, lingual; superior, middle, and inferior occipital), and parietal (inferior parietal and angular) regions $(p=0.043$ for all) in those who improved to $\mathrm{mRS} \leq 2$ at short-term follow-up PET, whereas SUVRg values remained unchanged in the $\mathrm{mRS}>2$ group (eFigure 3, links.lww.com/NXI/A695).

On exploratory SPM analysis, patients with LGI1-AE who improved to $\mathrm{mRS}<2$ showed increased cortical metabolic activity from baseline to follow-up, whereas those who had persistent disability $(\mathrm{mRS}>2)$ at the time of follow-up PET did not and, in fact, showed further worsening of cortical metabolism in some areas (Figure 5A).

\section{Relationship of Initial Metabolic Abnormalities With Long-Term Outcome in LGI1-AE}

At the median long-term follow-up of 19.7 months, $31 \%$ of the patients with LGI1-AE were asymptomatic, and of the remaining $69 \%$ who had residual symptoms, cognitive symptoms were present among all (Table 1 ). The median $\mathrm{mRS}$ reduced from 4 at the time of baseline PET to 1 at the time of their last clinical follow-up $(p=0.001)$ (eFigure 1, links.lww.com/NXI/A695). There was a nonsignificant trend for patients with long-term $\mathrm{mRS}>2$ to have longer time gap from symptom onset to baseline PET when compared with patients with $\mathrm{mRS} \leq 2$ (time [range]: 10.9 $[8.1-13.7]$ vs 2.3 [0.2-8.4] months; $p=0.051)$ (Figure 5C). 


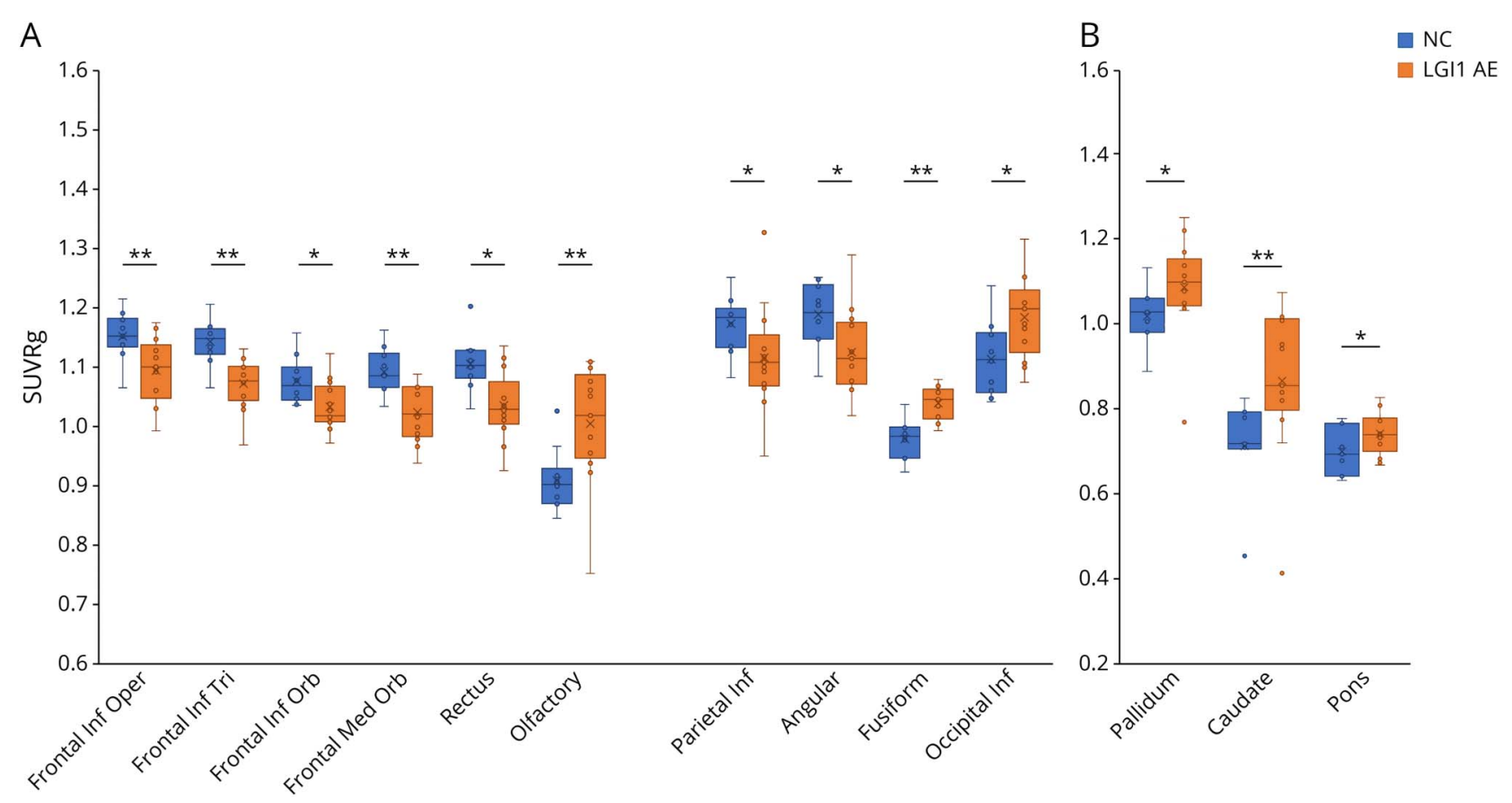

Comparison of brain metabolism in patients with LGI1-AE $(n=13)$ and NCS $(n=10)$ in $(A)$ cortical and (B) subcortical regions of interest. $* p<0.05$, $* * p<0.01$ (significant at the level of $p<0.05) .\left[{ }^{18} \mathrm{~F}\right] \mathrm{FDG}=\left[{ }^{18} \mathrm{~F}\right]$ fluorodeoxyglucose; $\mathrm{AE}=$ autoimmune encephalitis; Frontal Inf Oper = opercular part of inferior frontal gyrus; Frontal Inf Orb = orbital part of inferior frontal gyrus; Frontal Inf Tri = triangular part of inferior frontal gyrus; Frontal Med Orb = medial orbital part of superior frontal gyrus; LGI1 = leucine-rich, glioma-inactivated-1; NC = negative controls; occipital Inf; inferior occipital lobe; parietal Inf = inferior parietal lobe; SUVRg = global normalized standardized uptake value ratio

SPM analysis revealed that orbital frontal and cingulate gyrus metabolic activity was lower in the group of patients with worse long-term outcome $(\mathrm{mRS}>2)$ at the last clinical follow-up (peak uncorrected $p<0.01$ ) than in patients with better long-term outcome $(\mathrm{mRS} \leq 2)$ (Figure $5 \mathrm{~B})$. Moreover, on ROI analyses, baseline SUVRg was lower in anterior and middle cingulate in the long-term $\mathrm{mRS}>2$ group when compared with the mRS $\leq 2$ group $(-12.5 \%$ and $-6.9 \%$; $p=$ 0.026 for both) (eTable 8, links.lww.com/NXI/A695). No associations were observed between baseline mediotemporal or putaminal hypermetabolism and long-term clinical outcome (eTable 9, links.lww.com/NXI/A695).

\section{Classification of Evidence}

This study provides Class II evidence that semiquantitative measures of putaminal metabolism on PET can differentiate LGI1-AE group from non-LGI1-AE group, $\mathrm{AD}$ group, orNCs.

\section{Discussion}

Our study shows that semiquantitative FDG-PET indices of putaminal metabolism ( $\mathrm{P} / \mathrm{Mi}$ ratio and SUVRg) distinguished LGI1-AE group from non-LGI1-AE group, $\mathrm{AD}$ group, and NCs with high diagnostic accuracy. Furthermore, we reported the following in LGI1-AE group: (1) MTL-SUVRg associated with concurrent and short-term clinical disability changes; (2) widespread hypometabolism was seen in several neocortical regions, which improved in short term (approximately 6 months) among clinical responders; and (3) regional neocortical hypometabolism at initial presentation was associated with long-term clinical outcome in LGI-AE group.

Basal ganglia, hippocampal formation, and amygdala are among the regions with highest LGI1 expression in human brain (Consensus Human brain data set, Human Protein Atlas; available at proteinatlas.org). ${ }^{28-30}$ Anti-LGI1 antibodies have been shown to decrease expression of potassium (Kv1.1) channels on neuronal cell membranes, ${ }^{4}$ which in turn is associated with neuronal hyperexcitability and hypermetabolism. $\left[{ }^{18} \mathrm{~F}\right] \mathrm{FDG}$ concentrates in brain tissue in proportion to neuronal metabolic activity and is mediated by upregulation of glucose transporters and intracellular hexokinase enzyme, thus accounting for the hypermetabolic signal seen most prominently in putamen and MTL in anti-LGI1-AE patients.

Several previous studies have reported putaminal and mediotemporal hypermetabolism in LGI1-AE. ${ }^{9,14,17,19,31-33}$ Basal ganglia hypermetabolism was reported in $77 \%$ of patients with LGI1-AE and mediotemporal hypermetabolism in $62 \%$ of patients with LGI1-AE in a pooled cohort of 64 patients from 9 studies. ${ }^{9}$ However, only limited information is available about semiquantitative FDG-PET and its longitudinal implications in LGI1-AE. When compared with standard visual analyses, few studies have shown that semiquantitative 
Figure 4 Longitudinal Changes in Brain Metabolism After Immunosuppressive Treatment in Patients With LGI1-AE
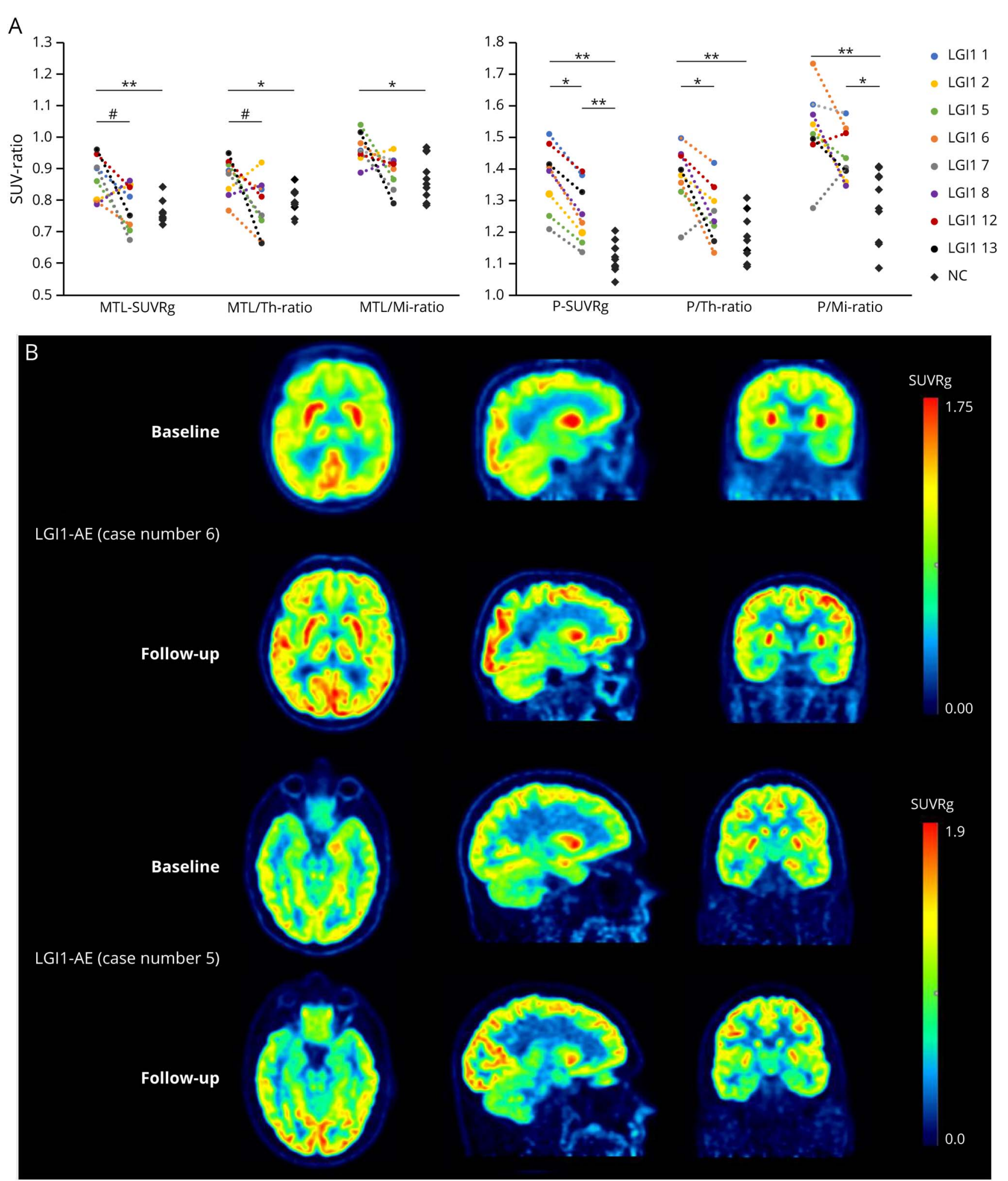

(A) Individual and group-level changes in brain metabolism from baseline to short-term follow-up in LGI1-AE group $(n=8)$, measured with $\left[{ }^{18} \mathrm{~F}\right] \mathrm{FDG}$ PET indices in mediotemporal lobe and putamen and compared with NCs $(n=10)$. (B) Visualization of individual changes in brain metabolism after immunotherapy in 2 patients with LGI1-AE using parametric [ $\left.{ }^{18} \mathrm{~F}\right]$ FDG SUVRg images. The images in (B) demonstrate decreases of the initial hypermetabolism in striatum (case 6) and mediotemporal lobe (case 5) and increases in occipital metabolism (both cases). The numbering of the LGI1 cases refers to the case numbers in Table 1. ${ }^{\star} p<0.05,{ }^{*} p<0.01$ (both significant at the level of $p<0.05$ ), $\# p=0.05$ (significant at the level of $p=0.05$ ). $\left[{ }^{18} \mathrm{~F}\right]$ FDG $=\left[{ }^{18} \mathrm{~F}\right]$ fluorodeoxyglucose; AE $=$ autoimmune encephalitis; $L G I 1=$ leucine-rich, glioma-inactivated-1; Mi = midbrain; $M T L=$ mediotemporal lobe; NC = negative control; $P=P$ Putamen; SUV = standardized uptake value; SUVRg = global brain normalized standardized uptake value ratio; Th = thalamus. 
A. $a m R S<2$
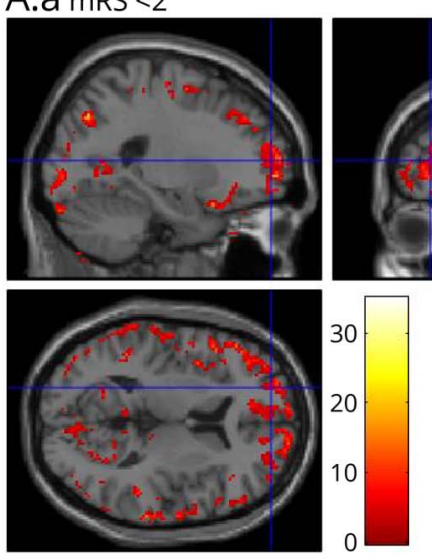

B
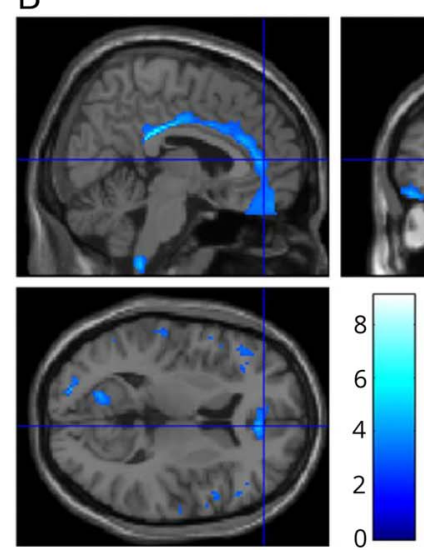

A. $b \mathrm{mRS}>2$
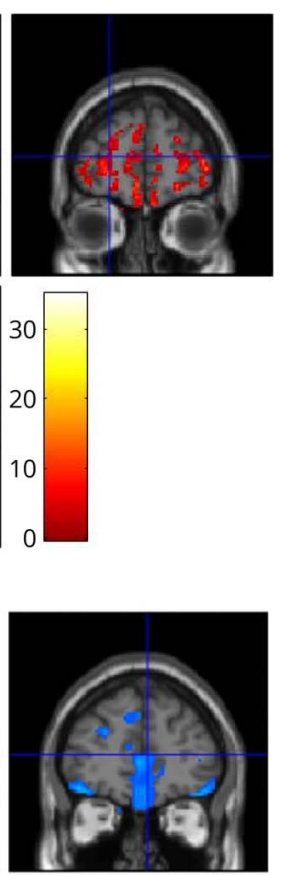

C
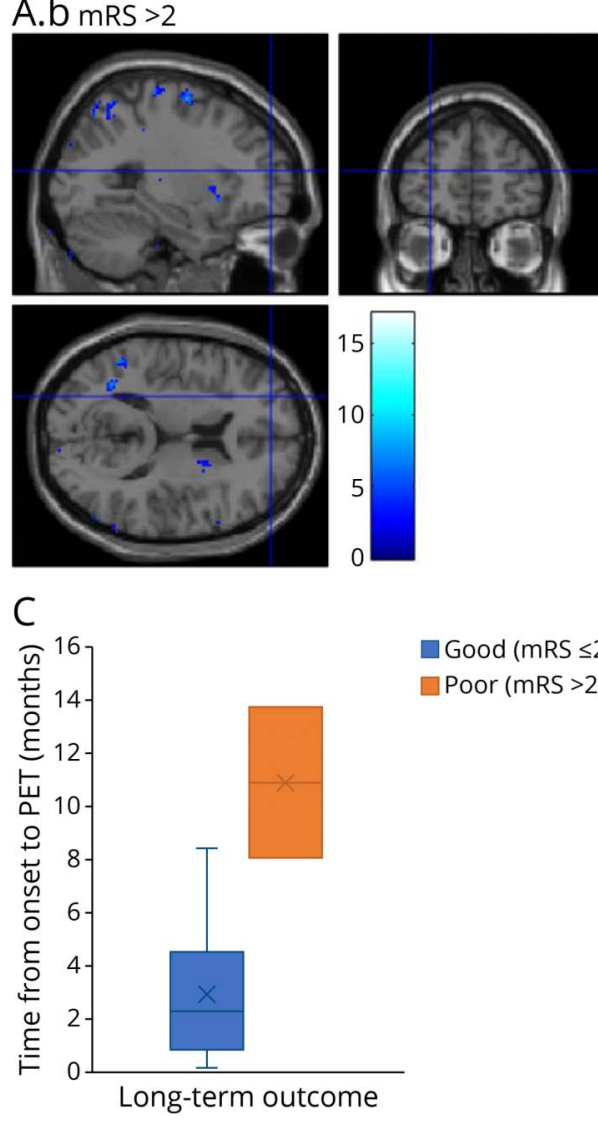

(A.a-b) Changes in brain metabolism from baseline to short-term followup in LGI1-AE group with no significant disability ( $\mathrm{mRS}<2, \mathrm{n}=3$ ) (A.a) and with moderate disability (mRS $>$ $2, n=3)(A \cdot b)$ as the short-term clinical outcome at the time of follow-up $\left[{ }^{18} \mathrm{~F}\right]$ FDG PET. Results from the statistical parametric mapping (SPM) analyses of voxel-wise [ $\left.{ }^{18} \mathrm{~F}\right]$ FDG SUVRg images are visualized with $T$ scores, with only voxels with significant within-group changes (threshold of $\mathrm{T}=2.92$ corresponding to uncorrected $p<0.05$ ) shown, as denoted by the color scale bar. Voxels with significant increases are shown for the mRS $<2$ group, and voxels with significant decreases are shown for the mRS > 2 group. (B) Differences in baseline brain metabolism in LGI1-AE group with good $(m R S \leq 2)$ vs poor ( $m R S>2$ ) long-term clinical outcome, visualized with voxel-level analysis of parametric $\left[{ }^{18} \mathrm{~F}\right] \mathrm{FDG}$ SUVRg images with SPM. The color scale bar represents voxellevel T scores, and only voxels with significant differences between groups (threshold of $\mathrm{T}=2.76$ corresponding to uncorrected $p<0.01$ ) are shown. (C) Differences in the time from symptom onset to the initial $\left[{ }^{18}\right.$ F]FDG-PET between LGI1-AE group with good $(m R S \leq 2)$ or poor $(m R S>2)$ long-term clinical outcome. $\left[{ }^{18} \mathrm{~F}\right.$ FDG $=$ $\left[{ }^{18}\right.$ Fffluorodeoxyglucose; $\mathrm{AE}=$ autoimmune encephalitis; Cingulum Post = posterior cingulum; LGI1 = leucine-rich, glioma-inactivated-1; Occipital Inf $=$ inferior occipital; Parietal Sup = superior parietal; SUVRg = global brain normalized standardized uptake value ratio. approaches with healthy control reference population further increase the sensitivity of FDG-PET to detect metabolic abnormalities in LGI1- $\mathrm{AE}^{9}$ or in cohorts of $\mathrm{AE}$ of mixed etiologies. $^{34,35}$ Moreover, $\mathrm{AD}$ occurs in an age group similar to that in LGI-AE, and visual analysis of FDG-PET images may be inadequate to distinguish $\mathrm{AD}$ from LGI-AE owing to relative cortical hypometabolism in $\mathrm{AD}$ that can give an appearance of an increased FDG-PET uptake in basal ganglia. To the best of our knowledge, our study is the first to systematically evaluate semiquantitative FDG-PET indices in LGI1-AE group compared with non-LGI1-AE and AD groups and provide long-term clinical outcome data in relation to FDG-PET in patients with LGI1-AE.

Although all semiquantitative indices yielded a high accuracy (AUC > 0.8), P/Mi yielded the highest accuracy for all group comparisons. From a mechanistic standpoint, midbrain may be an optimal reference region for FDG-PET analysis in LGI1-AE because it has very low expression of LGI1-RNA ${ }^{28}$ and may be least affected by the auto-aggressive LGI1 antibodies. We propose that a $\mathrm{P} / \mathrm{Mi}$ ratio cutoff value of $>1.44-1.46$ may be used to aid in early differential diagnosis of LGI1-AE. These cutoff values correspond to a positive likelihood ratio $>10$ and a negative likelihood ratio $<0.20$, emphasizing their high diagnostic accuracy, particularly in individuals with modest pretest probabilities of showing antiLGI-AE. $^{36}$ This approach could assist in clinical decisionmaking while awaiting serum and CSF antibody results that could take several days to return in clinical practice, ${ }^{16}$ and especially if conventional brain MRI finding shows negative results. $^{37}$ Although CSF-Tau, phospho-Tau, and amyloidbeta-42 evaluations may help distinguish $\mathrm{AD}$ from LGI1$\mathrm{AE},{ }^{38}$ FDG-PET suggestive of LGI1-AE in patients otherwise suspected of $\mathrm{AD}$ should trigger antibody evaluations. Finally, the use of our proposed exact cutoffs for brain FDG-PET requires future confirmation in larger cohorts and across PET scanners with variable reconstruction algorithms and image processing approaches.

We also showed that mediotemporal hypermetabolism did not help distinguish LGI1-AE from non-LGI1-AE and was present in both LGI1-AE and non-LGI1-AE groups when compared with NCs. However, within the anti-LGI1-AE group, MTL hypermetabolism associated with the severity of concurrent clinical disability during the acute phase. Moreover, a reduction in MTL hypermetabolism tracked the degree of clinical 
improvement at the time of follow-up PET (median 6 months from presentation). These findings emphasize the role of MTL pathology in manifestation of early clinical disability, likely mediated by the impact of MTL dysfunction on both seizure occurrence and cognitive impairment. Normalization of the mediotemporal hypermetabolism after immunotherapy in our LGI1-AE group at approximately 6 months follow-up is in concordance with the few earlier studies ${ }^{9,19}$ with follow-up intervals of 2-9 months. ${ }^{9,19}$ FDG-PET abnormalities in the acute phase of illness may represent an earlier pathophysiologic correlate of the long-term hippocampal atrophy seen in some patients with LGI1-AE. ${ }^{39}$ We also observed olfactory gyrus hypermetabolism in LGI1-AE. We hypothesize that the olfactory hypermetabolism may be functionally associated with mediotemporal hypermetabolism through the limbic system.

FDG-PET was more sensitive than MRI in detecting abnormalities in LGI-AE in our study, similar to previous studies. ${ }^{9,35}$ Furthermore, MTL hypermetabolism associated with the degree of clinical impairment at initial presentation. Thus, FDG-PET may provide clinically relevant information for detecting MTL dysfunction in LGI1-AE. Although useful to distinguish LGI1-AE group from NCs, semiquantitative evaluation of mediotemporal metabolism alone did not help distinguish LGI1-AE from other types of AE.

In addition to basal ganglia and MTL changes, we found significant hypometabolism in widespread cortical brain regions, particularly in inferior frontal and parietal lobes. Neocortical hypometabolism may be a result of functional impairment propagated along cortical and subcortical networks, arising from the sites of primary abnormalities in MTL and basal ganglia. ${ }^{29}$ Significant reductions in brain connectivity involving inferior frontal gyrus, anterior and posterior cingulate gyri, several regions of the default mode network (DMN), and higher visual networks have been previously demonstrated in patients with LGI1-AE using resting-state fMRI. ${ }^{29,30}$ Recovery of neocortical hypometabolism in short term after immunotherapy and its association with clinical improvement in our study supports a role of functional disconnection in the pathogenesis of this PET finding and also underscores their clinical relevance in the acute phase. On the other hand, occipital hypermetabolism may be a compensatory mechanism in response to MTL and DMN dysfunction in patients with LGI-AE during the acute/early phase of the illness. A similar pattern of occipital hypermetabolism has been reported as a compensatory phenomenon in patients with amnestic mild cognitive impairment. ${ }^{40}$ Moreover, while LGI1 is highly expressed in basal ganglia and hippocampal subfields, weak expression of LGIl is also reported in inner layers of cerebral cortex. ${ }^{29,41}$ Future studies using ultra-highresolution PET scanners may help delineate the extent of hypometabolism or localized hypermetabolism within cortical layers in patients with LGI1-AE, which may be undetectable on current PET scanners owing to their more limited spatial resolution.

Previous longitudinal studies have shown that long-term recovery of cognitive impairment lagged resolution of seizures ${ }^{8}$ in patients with LGI1-AE. Specifically, on long-term follow-up of more than 2 years, approximately $70 \%$ of patients with LGI1$\mathrm{AE}$ experienced mild-to-moderate residual cognitive impairment in a European cohort, ${ }^{8}$ similar to our study. Although a detailed neuropsychologic evaluation ${ }^{42}$ was not performed in our study, clinical disability at long-term follow-up in our cohort was primarily driven by cognitive symptoms. The trend for the association between longer delay from symptom onset to baseline PET and worse long-term outcome underlines the importance of prompt diagnosis in LGI1-AE. Of interest, reduced metabolic activity in limbic/paralimbic cortical regions at the time of presentation was associated with the ultimate mRS scores at long-term follow-up in our study (median duration 20 months). Mechanistically, this may mean that in some patients LGI1-AE, acute cortical hypometabolism may lead to more persistent structural injury and needs to be evaluated in future studies. Indeed, whole brain atrophy and gray matter reduction of glutamine/glutamate ratio (on magnetic resonance spectroscopy) have been reported on long-term follow-up (mean duration 33 months), ${ }^{43}$ but no previous data regarding long-term prognostic implications of FDG-PET are available in LGI-AE.

Another interesting finding in our study is that pallidum and caudate also showed increased metabolic activity when compared with NCs, although this is not often appreciated on visual analyses owing to a lower metabolic rate in the pallidum when compared with other basal ganglia structures.

We acknowledge several limitations to our study. Ours is a single-center study with limited sample sizes, and there is clearly a need for larger, prospective, multicenter FDG-PET imaging studies in LGI1-AE and other autoimmune encephalitides. ${ }^{44}$ Regarding using semiquantitative indices for differential diagnosis, apart from scanner differences, effects of previous treatment would need to be considered. Immunosuppressive treatment initiated before initial FDG-PET may be associated with false-negative $\mathrm{P} / \mathrm{Mi}$ values based on these cutoffs. In addition, we are reporting a case with APS showing striatal hypermetabolism and a high $\mathrm{P} / \mathrm{Mi}$ value of 1.57. Striatal hypermetabolism in systemic lupus erythematosus-associated chorea has been reported, ${ }^{45}$ but semiquantitative putaminal hypermetabolism in APS has not been reported earlier. Furthermore, neurologic conditions disproportionately affecting midbrain, such as progressive supranuclear palsy, ${ }^{46}$ may demonstrate high $\mathrm{P} / \mathrm{Mi}$ values because of low midbrain metabolism and should also be considered in differential diagnosis. FDGPET scans may be considered investigational ${ }^{44}$ and may not be readily available in all hospitals, and patients may require referral to a tertiary care center to undergo FDG-PET. Future studies should combine neuropsychologic testing with immunology, multimodality imaging including longitudinal FDG-PET, structural and functional MRI, and electrophysiologic measurements to further elucidate the pathophysiology of LGI-AE and to guide clinical diagnostic and therapeutic decision-making.

In conclusion, our data suggest that metabolic abnormalities in LGI1-AE extend beyond putamen and mediotemporal lobe into other subcortical and cortical regions and that 
semiquantitative FDG-PET may aid in differential diagnosis and longitudinal follow-up of patients with LGI1-AE. Further prospective, longitudinal, multicenter studies with larger patient populations are warranted to confirm these findings.

\section{Acknowledgment}

The authors thank Mr. Jon Hainer for his assistance in performing database searches.

\section{Study Funding}

E. Rissanen has received a Sigrid Jusélius Fellowship Grant. T. Singhal has received research funding from Nancy Davis Foundation's "Race to Erase MS" program, Ann Romney Center for Neurologic Diseases, Harvard Neuro-Discovery Center, National Multiple Sclerosis Society, Department of Defense, and Water Cove Charitable Foundation. Funding agencies did not have any role in performance of the research or preparation of the manuscript.

\section{Disclosure}

The authors report no conflicts of interest relevant to this work. Go to Neurology.org/NN for full disclosures.

\section{Publication History}

Received by Neurology: Neuroimmunology \& Neuroinflammation June 29, 2021. Accepted in final form December 14, 2021.

\section{Appendix Authors}

\begin{tabular}{|c|c|c|}
\hline Name & Location & Contribution \\
\hline $\begin{array}{l}\text { Eero Rissanen, } \\
\text { MD, PhD }\end{array}$ & $\begin{array}{l}\text { PET Imaging Program in } \\
\text { Neurologic Diseases, Ann } \\
\text { Romney Center for } \\
\text { Neurologic Diseases, } \\
\text { Department of Neurology, } \\
\text { Brigham and Women's } \\
\text { Hospital and Harvard } \\
\text { Medical School; Brigham } \\
\text { Multiple Sclerosis Center, } \\
\text { Ann Romney Center for } \\
\text { Neurologic Diseases, } \\
\text { Department of Neurology, } \\
\text { Brigham and Women's } \\
\text { Hospital and Harvard } \\
\text { Medical School }\end{array}$ & $\begin{array}{l}\text { Drafting/revision } \\
\text { of the article for } \\
\text { content, including } \\
\text { medical writing for } \\
\text { content; major } \\
\text { role in the } \\
\text { acquisition of } \\
\text { data; and analysis } \\
\text { or interpretation } \\
\text { of data }\end{array}$ \\
\hline $\begin{array}{l}\text { Kelsey Carter, } \\
\text { MSN, CNP }\end{array}$ & $\begin{array}{l}\text { PET Imaging Program in } \\
\text { Neurologic Diseases, Ann } \\
\text { Romney Center for } \\
\text { Neurologic Diseases, } \\
\text { Department of Neurology, } \\
\text { Brigham and Women's } \\
\text { Hospital and Harvard } \\
\text { Medical School }\end{array}$ & $\begin{array}{l}\text { Drafting/revision } \\
\text { of the article for } \\
\text { content, including } \\
\text { medical writing for } \\
\text { content; major } \\
\text { role in the } \\
\text { acquisition of } \\
\text { data; and analysis } \\
\text { or interpretation } \\
\text { of data }\end{array}$ \\
\hline Steven Cicero, BS & $\begin{array}{l}\text { PET Imaging Program in } \\
\text { Neurologic Diseases, Ann } \\
\text { Romney Center for } \\
\text { Neurologic Diseases, } \\
\text { Department of Neurology, } \\
\text { Brigham and Women's } \\
\text { Hospital and Harvard } \\
\text { Medical School }\end{array}$ & $\begin{array}{l}\text { Drafting/revision } \\
\text { of the article for } \\
\text { content, including } \\
\text { medical writing for } \\
\text { content; major } \\
\text { role in the } \\
\text { acquisition of } \\
\text { data; and analysis } \\
\text { or interpretation } \\
\text { of data }\end{array}$ \\
\hline
\end{tabular}

Appendix (continued)

\begin{tabular}{|c|c|c|}
\hline Name & Location & Contribution \\
\hline John Ficke, BA & $\begin{array}{l}\text { PET Imaging Program in } \\
\text { Neurologic Diseases, Ann } \\
\text { Romney Center for } \\
\text { Neurologic Diseases, } \\
\text { Department of } \\
\text { Neurology, Brigham } \\
\text { and Women's } \\
\text { Hospital and } \\
\text { Harvard Medical } \\
\text { School }\end{array}$ & $\begin{array}{l}\text { Drafting/revision } \\
\text { of the article for } \\
\text { content, including } \\
\text { medical writing for } \\
\text { content, and } \\
\text { major role in the } \\
\text { acquisition of data }\end{array}$ \\
\hline
\end{tabular}

\begin{tabular}{|c|c|c|}
\hline $\begin{array}{l}\text { Marie Kijewski, } \\
\text { DSc }\end{array}$ & $\begin{array}{l}\text { Division of Nuclear } \\
\text { Medicine and Molecular } \\
\text { Imaging (M.K.), } \\
\text { Department of Radiology, } \\
\text { Brigham and Women's } \\
\text { Hospital and Harvard } \\
\text { Medical School, Boston, } \\
\text { MA }\end{array}$ & $\begin{array}{l}\text { Drafting/revision } \\
\text { of the article for } \\
\text { content, including } \\
\text { medical writing for } \\
\text { content, and } \\
\text { analysis or } \\
\text { interpretation of } \\
\text { data }\end{array}$ \\
\hline Mi-Ae Park, PhD & $\begin{array}{l}\text { Medical Physics Section, } \\
\text { Radiology Department, } \\
\text { University of Texas } \\
\text { Southwestern Medical } \\
\text { Center, Dallas, TX }\end{array}$ & $\begin{array}{l}\text { Drafting/revision } \\
\text { of the article for } \\
\text { content, including } \\
\text { medical writing for } \\
\text { content, and } \\
\text { analysis or } \\
\text { interpretation of } \\
\text { data }\end{array}$ \\
\hline
\end{tabular}

Joseph Kijewski, Department of Neurology, Drafting/revision
BS Brigham and Women's of the article for content, including medical writing for content, and major role in the acquisition of data

\begin{tabular}{|c|c|c|}
\hline Emily Stern, MD & $\begin{array}{l}\text { Ceretype Neuromedicine, } \\
\text { Cambridge, MA }\end{array}$ & $\begin{array}{l}\text { Drafting/revision } \\
\text { of the article for } \\
\text { content, including } \\
\text { medical writing for } \\
\text { content, and } \\
\text { Analysis or } \\
\text { interpretation of } \\
\text { data }\end{array}$ \\
\hline $\begin{array}{l}\text { Tanuja Chitnis, } \\
\text { MD }\end{array}$ & $\begin{array}{l}\text { Brigham Multiple } \\
\text { Sclerosis Center, Ann } \\
\text { Romney Center for } \\
\text { Neurologic Diseases, } \\
\text { Department of Neurology, } \\
\text { Brigham and Women's } \\
\text { Hospital and Harvard } \\
\text { Medical School, Boston, } \\
\text { MA }\end{array}$ & $\begin{array}{l}\text { Drafting/revision } \\
\text { of the article for } \\
\text { content, including } \\
\text { medical writing for } \\
\text { content, and } \\
\text { analysis or } \\
\text { interpretation of } \\
\text { data }\end{array}$ \\
\hline $\begin{array}{l}\text { David } \\
\text { Silbersweig, MD }\end{array}$ & $\begin{array}{l}\text { Functional Neuroimaging } \\
\text { Laboratory, Department } \\
\text { of Psychiatry, Brigham } \\
\text { and Women's Hospital } \\
\text { and Harvard Medical } \\
\text { School, Boston, MA }\end{array}$ & $\begin{array}{l}\text { Drafting/revision } \\
\text { of the article } \\
\text { for content, } \\
\text { including } \\
\text { medical writing } \\
\text { for content; } \\
\text { Analysis or } \\
\text { interpretation of } \\
\text { data }\end{array}$ \\
\hline $\begin{array}{l}\text { Howard L. } \\
\text { Weiner, MD }\end{array}$ & $\begin{array}{l}\text { Brigham Multiple } \\
\text { Sclerosis Center, Ann } \\
\text { Romney Center for } \\
\text { Neurologic Diseases, } \\
\text { Department of Neurology, } \\
\text { Brigham and Women's } \\
\text { Hospital and Harvard } \\
\text { Medical School, Boston, } \\
\text { MA }\end{array}$ & $\begin{array}{l}\text { Drafting/revision } \\
\text { of the article for } \\
\text { content, including } \\
\text { medical writing for } \\
\text { content, and } \\
\text { analysis or } \\
\text { interpretation of } \\
\text { data }\end{array}$ \\
\hline
\end{tabular}


Appendix (continued)

\begin{tabular}{ll}
\hline Name & Location \\
\hline Chun K. Kim, MD & Nuclear Medicine, \\
& Department of Medicine, \\
& College of Medicine, \\
& Hanyang University, \\
& Seoul, Republic of Korea
\end{tabular}

Drafting/revision

of the article for

content, including

medical writing for

content; major

role in the

acquisition of

data; Study

concept or design;

and analysis or

interpretation of

\begin{tabular}{ll}
\hline Jennifer Lyons, & Biogen Inc., Cambridge, \\
MD & MA
\end{tabular}

Drafting/revision

of the article for

content, including

medical writing for

content; major

role in the

acquisition of

data; study

concept or design;

and analysis or

interpretation of

data

\section{Joshua P. Klein \\ MD, PhD}

Department of Neurology,

Brigham and Women's

Hospital and Harvard

Medical School, Boston, MA
Drafting/revision

of the article for content, including medical writing for content; major role in the acquisition of data; study concept or design; and analysis or interpretation of data

\begin{tabular}{ll}
\hline Shamik & Brigham Multiple \\
Bhattacharyya, & Sclerosis Center, Ann \\
MD, MS & Romney Center for \\
& Neurologic Diseases, \\
& Department of Neurology, \\
& Brigham and Women's \\
& Hospital and Harvard \\
& Medical School, Boston, \\
& MA
\end{tabular}

Drafting/revision of the article for content, including medical writing for content; major role in the acquisition of data; study concept or design; and analysis or interpretation of data

\section{Tarun Singhal, MD, MBBS}

\begin{tabular}{|c|c|}
\hline $\begin{array}{l}\text { PET Imaging Program in } \\
\text { Neurologic Diseases, Ann } \\
\text { Romney Center for } \\
\text { Neurologic Diseases, } \\
\text { Department of Neurology, } \\
\text { Brigham and Women's } \\
\text { Hospital and Harvard } \\
\text { Medical School, Boston, } \\
\text { MA; Brigham Multiple } \\
\text { Sclerosis Center, Ann } \\
\text { Romney Center for } \\
\text { Neurologic Diseases, } \\
\text { Department of Neurology, } \\
\text { Brigham and Women's } \\
\text { Hospital and Harvard } \\
\text { Medical School, Boston, } \\
\text { MA }\end{array}$ & $\begin{array}{l}\text { Drafting/revision } \\
\text { of the article for } \\
\text { content, including } \\
\text { medical writing for } \\
\text { content; major } \\
\text { role in the } \\
\text { acquisition of } \\
\text { data; study } \\
\text { concept or design; } \\
\text { and analysis or } \\
\text { interpretation of } \\
\text { data }\end{array}$ \\
\hline
\end{tabular}

\section{References}

1. Leypoldt F, Armangue T, Dalmau J. Autoimmune encephalopathies. Ann N Y Acad Sci. 2015;1338(1):94-114

2. Alexopoulos H, Dalakas MC. The immunobiology of autoimmune encephalitides. J Autoimmun. 2019;104:102339.
3. Irani SR, Alexander S, Waters P, et al. Antibodies to Kvl potassium channel-complex proteins leucine-rich, glioma inactivated 1 protein and contactin-associated protein-2 in limbic encephalitis, Morvan's syndrome and acquired neuromyotonia. Brain. 2010; 133(9):2734-2748

4. Petit-Pedrol M, Sell J, Planaguma J, et al. LGI1 antibodies alter Kv1.1 and AMPA receptors changing synaptic excitability, plasticity and memory. Brain. 2018;141(11): 3144-3159.

5. Lai M, Huijbers MG, Lancaster E, et al. Investigation of LGI1 as the antigen in limbic encephalitis previously attributed to potassium channels: a case series. Lancet Neurol. 2010;9(8):776-785.

6. Irani SR, Michell AW, Lang B, et al. Faciobrachial dystonic seizures precede Lgil antibody limbic encephalitis. Ann Neurol. 2011;69(5):892-900.

7. Andrade DM, Tai P, Dalmau J, Wennberg R. Tonic seizures: a diagnostic clue of antiLGI1 encephalitis? Neurology. 2011;76(15):1355-1357.

8. van Sonderen A, Thijs RD, Coenders EC, et al. Anti-LGI1 encephalitis: clinical syndrome and long-term follow-up. Neurology. 2016;87(14):1449-1456.

9. Liu X, Shan W, Zhao X, et al. The clinical value of (18) F-FDG-PET in autoimmune encephalitis associated with LGI1 antibody. Front Neurol. 2020;11:418.

10. Irani SR, Stagg CJ, Schott JM, et al. Faciobrachial dystonic seizures: the influence of immunotherapy on seizure control and prevention of cognitive impairment in a broadening phenotype. Brain. 2013;136(pt 10):3151-3162.

11. Thompson J, Bi M, Murchison AG, et al. The importance of early immunotherapy in patients with faciobrachial dystonic seizures. Brain. 2018;141(2):348-356.

12. Baumgartner A, Rauer S, Hottenrott $\mathrm{T}$, et al. Admission diagnoses of patients later diagnosed with autoimmune encephalitis. J Neurol. 2019;266(1):124-132.

13. Marquetand J, van Lessen M, Bender B, et al. Slowly progressive LGI1 encephalitis with isolated late-onset cognitive dysfunction: a treatable mimic of Alzheimer's disease. Eur J Neurol. 2016;23(5):e28-29.

14. Shin YW, Lee ST, Shin JW, et al. VGKC-complex/LGI1-antibody encephalitis: clinical manifestations and response to immunotherapy. J Neuroimmunol. 2013; 265(1-2):75-81.

15. Jarius S, Hoffmann L, Clover L, Vincent A, Voltz R. CSF findings in patients with voltage gated potassium channel antibody associated limbic encephalitis. J Neurol Sci. 2008;268(1-2):74-77.

16. Galetta K, Gheihman G, Rosen A, Klein JP, Bhattacharyya S. Influence of autoimmune antibody testing on the use of immunotherapy on an inpatient Neurology service. Neurohospitalist. 2021;11(3):214-220.

17. Tripathi M, Tripathi M, Roy SG, et al. Metabolic topography of autoimmune nonparaneoplastic encephalitis. Neuroradiology. 2018;60(2):189-198.

18. Wegner F, Wilke F, Raab P, et al. Anti-leucine rich glioma inactivated 1 protein and anti-N-methyl-D-aspartate receptor encephalitis show distinct patterns of brain glucose metabolism in 18F-fluoro-2-deoxy-d-glucose positron emission tomography. BMC Neurol. 2014;14:136.

19. Navarro V, Kas A, Apartis E, et al. Motor cortex and hippocampus are the two main cortical targets in LGI1-antibody encephalitis. Brain. 2016;139(pt 4):1079-1093.

20. Graus F, Titulaer MJ, Balu R, et al. A clinical approach to diagnosis of autoimmune encephalitis. Lancet Neurol. 2016;15(4):391-404.

21. Schubert J, Bramer D, Huttner HB, et al. Management and prognostic markers in patients with autoimmune encephalitis requiring ICU treatment. Neurol Neuroimmunol Neuroinflamm. 2019;6(1):e514.

22. Tzourio-Mazoyer N, Landeau B, Papathanassiou D, et al. Automated anatomical labeling of activations in SPM using a macroscopic anatomical parcellation of the MNI MRI single-subject brain. NeuroImage. 2002;15(1):273-289.

23. Waxman $\mathrm{AD}$, Herholz $\mathrm{K}$, Lewis $\mathrm{DH}$, et al. Society of nuclear medicine procedure guideline for FDG PET brain imaging [online]. Accessed October 29, 2020. s3. amazonaws.com/rdcms-snmmi/files/production/public/docs/Society $\% 20$ of $\%$ 20Nuclear\%20Medicine\%20Procedure\%20Guideline\%20for\%20FDG\%20PET\% 20Brain\%20Imaging.pdf.

24. Minoshima S, Frey KA, Koeppe RA, Foster NL, Kuhl DE. A diagnostic approach in Alzheimer's disease using three-dimensional stereotactic surface projections of fluorine-18-FDG PET. J Nucl Med. 1995;36(7):1238-1248.

25. Berti V, Mosconi L, Pupi A. Brain: normal variations and benign findings in fluorodeoxyglucose-PET/computed tomography imaging. PET Clin. 2014;9(2):129-140.

26. DeLong ER, DeLong DM, Clarke-Pearson DL. Comparing the areas under two or more correlated receiver operating characteristic curves: a nonparametric approach Biometrics. 1988;44(3):837-845.

27. Youden WJ. Index for rating diagnostic tests. Cancer. 1950;3(1):32-35.

28. Uhlen M, Fagerberg L, Hallstrom BM, et al. Proteomics. Tissue-based map of the human proteome. Science. 2015;347(6220):1260419.

29. Heine J, Pruss H, Kopp UA, et al. Beyond the limbic system: disruption and functional compensation of large-scale brain networks in patients with anti-LGI1 encephalitis. J Neurol Neurosurg Psychiatry. 2018;89(11):1191-1199.

30. Qiao J, Zhao X, Wang S, et al. Functional and structural brain alterations in encephalitis with LGI1 antibodies. Front Neurosci. 2020;14:304.

31. Probasco JC, Solnes L, Nalluri A, et al. Abnormal brain metabolism on FDG-PET/CT is a common early finding in autoimmune encephalitis. Neurol Neuroimmunol Neuroinflamm. 2017;4(4):e352.

32. Solnes LB, Jones KM, Rowe SP, et al. Diagnostic value of (18)F-FDG PET/CT versus MRI in the setting of antibody-specific autoimmune encephalitis. J Nucl Med. 2017; 58(8):1307-1313.

33. Celicanin M, Blaabjerg M, Maersk-Moller C, et al. Autoimmune encephalitis associated with voltage-gated potassium channels-complex and leucine-rich gliomainactivated 1 antibodies_a national cohort study. Eur J Neurol. 2017;24(8):999-1005. 
34. Lv RJ, Pan J, Zhou G, et al. Semi-quantitative FDG-PET analysis increases the sensitivity compared with visual analysis in the diagnosis of autoimmune encephalitis. Front Neurol. 2019;10:576.

35. Moreno-Ajona D, Prieto E, Grisanti F, et al. F-FDG-PET imaging patterns in autoimmune encephalitis: impact of image analysis on the results. Diagnostics (Basel). 1820;10(6):356.

36. Deeks JJ, Altman DG. Diagnostic tests 4: likelihood ratios. BMJ. 2004;329(7458): 168-169.

37. Abboud H, Probasco JC, Irani S, et al. Autoimmune encephalitis: proposed best practice recommendations for diagnosis and acute management. J Neurol Neurosurg Psychiatry. 2021;92(7):757-768.

38. Lardeux P, Fourier A, Peter E, et al. Core cerebrospinal fluid biomarker profile in antiLGI1 encephalitis. J Neurol. 2022;269:377-388.

39. Hanert A, Rave J, Granert O, et al. Hippocampal dentate gyrus atrophy predicts pattern separation impairment in patients with LGI1 encephalitis. Neuroscience. 2019;400:120-131.

40. Ashraf A, Fan Z, Brooks DJ, Edison P. Cortical hypermetabolism in MCI subjects: a compensatory mechanism?. Eur J Nucl Med Mol Imaging. 2015;42(3):447-458.
41. Herranz-Perez V, Olucha-Bordonau FE, Morante-Redolat JM, Perez-Tur J. Regional distribution of the leucine-rich glioma inactivated (LGI) gene family transcripts in the adult mouse brain. Brain Res. 2010;1307:177-194.

42. Griffith SP, Malpas CB, Alpitsis R, O’Brien TJ, Monif M. The neuropsychological spectrum of anti-LGI1 antibody mediated autoimmune encephalitis. J Neuroimmunol. 2020;345:577271.

43. Szots M, Blaabjerg M, Orsi G, et al. Global brain atrophy and metabolic dysfunction in LGI1 encephalitis: a prospective multimodal MRI study. J Neurol Sci. 2017;376: 159-165.

44. Morbelli S, Arbizu J, Booij J, et al. The need of standardization and of large clinical studies in an emerging indication of [(18)F]FDG PET: the autoimmune encephalitis. Eur J Nucl Med Mol Imaging. 2017;44(3):353-357.

45. Krakauer M, Law I. FDG PET brain imaging in neuropsychiatric systemic lupus erythematosis with choreic symptoms. Clin Nucl Med. 2009;34(2):122-123.

46. Mishina M, Ishii K, Mitani K, et al. Midbrain hypometabolism as early diagnostic sign for progressive supranuclear palsy. Acta Neurol Scand. 2004;110(2):128-135. 


\section{Neurology \\ Neuroimmunology \& Neuroinflammation}

\section{Cortical and Subcortical Dysmetabolism Are Dynamic Markers of Clinical Disability and Course in Anti-LGI1 Encephalitis \\ Eero Rissanen, Kelsey Carter, Steven Cicero, et al. \\ Neurol Neuroimmunol Neuroinflamm 2022;9; \\ DOI 10.1212/NXI.0000000000001136}

This information is current as of January 28, 2022

Updated Information \&

Services

References

Subspecialty Collections

Permissions \& Licensing

Reprints including high resolution figures, can be found at:

http://nn.neurology.org/content/9/2/e1136.full.html

This article cites 45 articles, 7 of which you can access for free at: http://nn.neurology.org/content/9/2/e1136.full.html\#\#ref-list-1

This article, along with others on similar topics, appears in the following collection(s):

Class III

http://nn.neurology.org//cgi/collection/class_iii

PET

http://nn.neurology.org//cgi/collection/pet

Information about reproducing this article in parts (figures,tables) or in its entirety can be found online at:

http://nn.neurology.org/misc/about.xhtml\#permissions

Information about ordering reprints can be found online:

http://nn.neurology.org/misc/addir.xhtml\#reprintsus

Neurol Neuroimmunol Neuroinflamm is an official journal of the American Academy of Neurology.

Published since April 2014, it is an open-access, online-only, continuous publication journal. Copyright

Copyright (C) 2022 The Author(s). Published by Wolters Kluwer Health, Inc. on behalf of the American

Academy of Neurology.. All rights reserved. Online ISSN: 2332-7812.

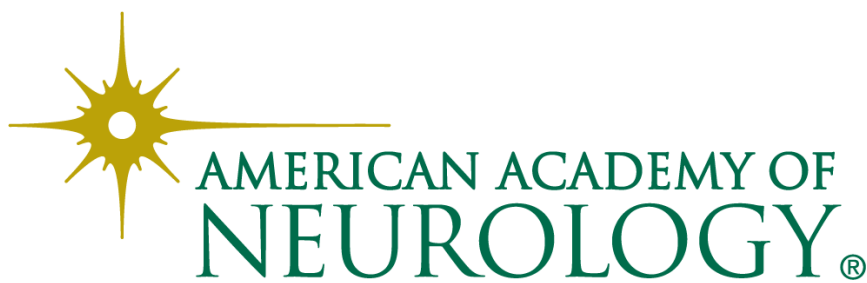

